\title{
Small Molecules Engage Hot Spots through Cooperative Binding To Inhibit a Tight Protein-Protein Interaction
}

\author{
${ }_{3}$ Degang Liu, ${ }^{\dagger}$ David Xu, ${ }^{\S} \|^{\prime}$ Min Liu, ${ }^{\S}$ William Eric Knabe, ${ }^{\dagger}$ Cai Yuan, ${ }^{\S}$ Donghui Zhou, ${ }^{\dagger}$ \\ ${ }_{4}$ Mingdong Huang, ${ }^{\S}$ and Samy O. Meroueh ${ }^{*},+\neq(0$ \\ $5{ }^{\dagger}$ Department of Biochemistry and ${ }^{*}$ Department of Molecular Biology, Center for Computational Biology and Bioinformatics, Indiana \\ 6 University School of Medicine, Indianapolis, Indiana 46202, United States \\ $7{ }^{\S}$ Fujian Institute of Research on the Structure of Matter, Chinese Academy of Science, Gulou District, Fuzhou, Fujian 350002, China \\ 8 "Department of BioHealth Informatics, Indiana University School of Informatics and Computing, Indianapolis, Indiana 46202, United \\ 9 States
}

11 ABSTRACT: Protein-protein interactions drive every aspect 12 of cell signaling, yet only a few small-molecule inhibitors of 13 these interactions exist. Despite our ability to identify critical 14 residues known as hot spots, little is known about how to 15 effectively engage them to disrupt protein-protein inter16 actions. Here, we take advantage of the ease of preparation and 17 stability of pyrrolinone 1 , a small-molecule inhibitor of the 18 tight interaction between the urokinase receptor (uPAR) and 19 its binding partner, the urokinase-type plasminogen activator $20 \mathrm{uPA}$, to synthesize more than 40 derivatives and explore their 21 effect on the protein-protein interaction. We report the 22 crystal structure of UPAR bound to previously discovered

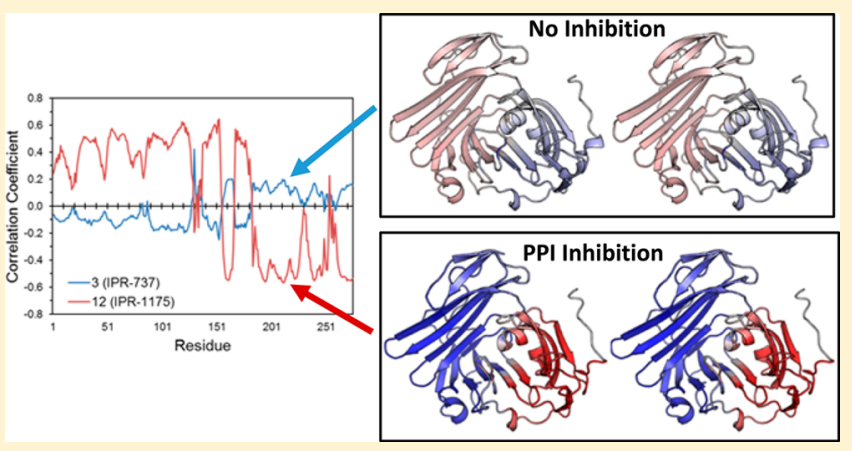
pyrazole 3 and to pyrrolinone 12. While both 3 and 12 bind to UPAR and compete with a fluorescently labeled peptide probe, only 12 and its derivatives inhibit the full uPAR·uPA interaction. Compounds 3 and 12 mimic and engage different hot-spot residues on uPA and $\mathrm{uPAR}$, respectively. Interestingly, 12 is involved in a $\pi$-cation interaction with Arg-53, which is not considered a hot spot. Explicit-solvent molecular dynamics simulations reveal that 3 and 12 exhibit dramatically different correlations of motion with residues on uPAR. Free energy calculations for the wild-type and mutant uPAR bound to uPA or 12 show that Arg-53 interacts with uPA or with 12 in a highly cooperative manner, thereby altering the contributions of hot spots to uPAR binding. The direct engagement of peripheral residues not considered hot spots through $\pi$-cation or salt-bridge interactions could provide new opportunities for enhanced small-molecule engagement of hot spots to disrupt challenging protein-protein interactions.

$32 \mathrm{~T}$ is estimated that there are more than 200000 protein33 protein interactions in a cell. These interactions drive nearly 34 every aspect of cellular function. Small molecules offer an 35 opportunity to explore these interactions in normal and 36 pathological processes. Protein-protein interactions exhibit a 37 wide range of binding thermodynamics and kinetics from weak 38 interactions that occur over small interfaces to tight and stable 39 protein-protein interactions that occur over large interfaces $40\left(>1000 \AA^{2}\right)$. Although the design of small molecules to disrupt 41 weak interactions is relatively straightforward, inhibiting tight 42 interactions is much more challenging. To date, while 43 numerous small molecules have been reported to inhibit 44 protein-protein interactions (reviewed in refs 1 and 2), there 45 are only a few examples of small molecules that disrupt tight 46 protein-protein interactions $\left(K_{\mathrm{d}}=1-100 \mathrm{nM}\right)$; examples 47 include antagonists of $\mathrm{Bcl}-\mathrm{xL}^{3}$ and IL-2R ${ }^{4}$ The ability to disrupt 48 these tight interactions that occur over large surfaces with a 49 small molecule that has a much smaller footprint is attributed to the presence of residues that contribute disproportionately to 50 the binding affinity, also known as hot spots. ${ }^{5-9} 51$

The urokinase receptor (uPAR) is a cell surface glyco- 52 phosphatidylinositol (GPI)-anchored receptor that is part of an 53 extensive network of protein-protein interactions. Its binding 54 partners include serine proteinase urokinase-type plasminogen 55 activator $\mathrm{uPA}^{10}$ and the glycoprotein vitronectin. ${ }^{11-13}$ The 56 $\mathrm{uPAR} \cdot \mathrm{uPA}$ protein-protein interaction is a high-affinity $\left(K_{\mathrm{D}}=57\right.$ $1 \mathrm{nM})$ and stable $\left(k_{\text {off }}=10^{-4} \mathrm{~s}^{-1}\right)$ interaction. ${ }^{14}$ Crystal 58 structures of the uPAR.uPA complex show that uPA binds to 59 UPAR along a well-defined binding site that is part of a $>100060$ $\AA^{2}$ interface. ${ }^{15-18}$ The interaction is mediated by a 25 -residue 61 growth factor-like domain (GFD), and residues from a kringle- 62 like domain of uPA. A comprehensive alanine scanning study at 63

Received: October 10, 2016

Revised: February 2, 2017

Published: February 10, 2017 
64 the uPAR $\cdot u P A$ interface identified several hot-spot residues that 65 reduced the uPAR $\cdot u P A$ binding affinity by $>1 \mathrm{kcal} \mathrm{mol}^{-1}$. 14,18,19 66 In the uPAR-uPA complex, there are hot spots both on uPA 67 (Trp-30, Phe-25, Tyr-24, and Ile-28) ${ }^{14}$ and on the large binding 68 cleft of uPAR that accommodates the GFD domain (Leu-150, 69 Leu-55, Leu-66, Tyr-57, and Asp-140). ${ }^{18}$

70 Previously, we performed a computational screen of 71 commercial libraries to identify small molecules that bind to $72 \mathrm{UPAR}$ and disrupt the uPAR.uPA protein-protein interac73 tion. $^{18,21,22}$ Compound $\mathbf{1}$ was discovered following a sub74 structure search using pyrazole, piperidinone, and pyrrolydi75 none compounds as templates. ${ }^{23}$ These compounds were 76 shown to bind to uPAR and displace a fluorescently labeled 77 peptide. In addition to 1 , another strategy that consisted of 78 docking chemical libraries to an ensemble of uPAR structures 79 sampled from explicit-solvent molecular dynamics simulations 80 led to 2 (IPR-803). ${ }^{24}$ Compound 2 binds to UPAR with 81 submicromolar affinity and inhibits the uPAR.uPA complex 82 with single-digit micromolar $\mathrm{IC}_{50}$ values. ${ }^{24}$ Structure-activity 83 relationships revealed the critical nature of a benzoic acid 84 moiety that was attributed to a salt-bridge interaction with the 85 guanidinium group of Arg-53. We showed that this residue 86 became exposed following explicit-solvent molecular dynamics 87 simulations. ${ }^{16}$ Our predicted binding mode and interaction 88 with Arg-53 was recently independently confirmed by a crystal 89 structure of an analogue of $\mathbf{2}$ bound to uPAR. ${ }^{25}$ To the best of 90 our knowledge, our work with $\mathbf{2}$ is the first study that led to 91 small-molecule protein-protein antagonists using protein 92 structures sampled from molecular dynamics simulations. The 93 work highlights the importance of considering molecular 94 dynamics of a receptor for the design of small molecules that 95 disrupt tight interactions (Scheme 1).

\section{Scheme 1}

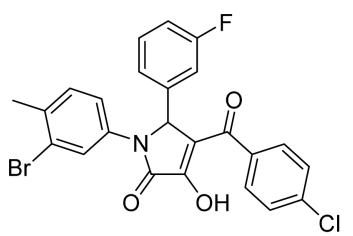

1 (IPR-1110)

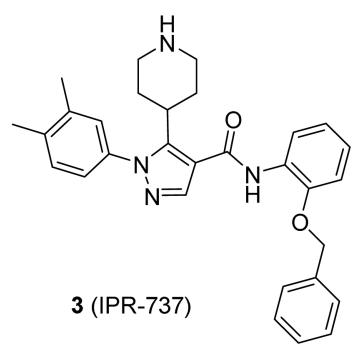

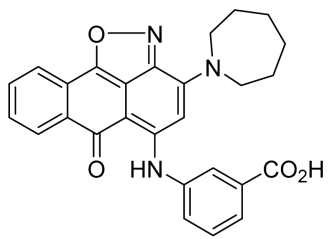

2 (IPR-803)
96 Analysis of three-dimensional structures of protein-protein 97 complexes reveals that hot spots are generally found at the 98 protein-protein interface of tight protein-protein interac99 tions. $^{5,26,27}$ They can be located either on the ligand or on the 100 receptor that harbors a binding site. It has been suggested that 101 small molecules that bind to hot spots can disrupt tight 102 protein-protein interactions despite their smaller footprint. 103 Early work targeting the IL-2-IL-2R $\alpha$ complex confirmed that 104 small molecules that inhibited the interaction directly bind to hot spots, ${ }^{28}$ but the researchers also found that neighboring 105 residues that promote IL-2.IL-2R $\alpha$ enhanced binding of small 106 molecules. These counterintuitive results can be explained by 107 dynamical effects likely arising from the cooperativity of 108 residues at the interface. ${ }^{29}$ This cooperativity has led to the 109 suggestion that hot-spot clusters can form hot regions. ${ }^{30} 110$ Cooperativity makes it more difficult to select the appropriate 111 hot spots for the rational design of small-molecule inhibitors of 112 protein-protein interactions. The mere binding to hot spots 113 may not necessarily result in more potent inhibition of the 114 protein-protein interaction. A deeper understanding of (i) the 115 interaction energies between small molecules and individual 116 residues and (ii) the dynamical changes that occur upon 117 binding of a small molecule could lead to more effective 118 strategies for the rational design of small-molecule protein- 119 protein interaction inhibitors.

120

Here, we take advantage of the straightforward synthesis of $\mathbf{1} 121$ to prepare 46 derivatives of the compound to gain insight into 122 the forces that lead to small-molecule inhibition of the UPAR 123 uPA protein-protein interaction. Their activity was measured 124 using fluorescence polarization with a labeled $\alpha$-helical peptide 125 and an enzyme-linked immunosorbent assay (ELISA) that uses 126 $\mathrm{uPA}_{\mathrm{ATF}}$, which includes the entire binding interface of the 127 uPAR.uPA interface. We determine the crystal structure of 128 uPAR bound to two small molecules, namely, pyrrolinone 21129 (IPR-1175) and pyrrazole 3 (IPR-737), providing a structural 130 basis for the activity of $\mathbf{1}$ and its derivatives. To gain deeper 131 insight into the basis for the activity of $\mathbf{1}$ and its derivatives, we 132 conducted explicit-solvent molecular dynamics simulations of 133 UPAR in complex with uPA, 1, 3, and several other derivatives. 134 The resulting structures were used for free energy calculations 135 using molecular mechanics (MM), generalized Born (GB), and 136 solvent-accessible surface (SA) also known as MM-GBSA. 137 Decomposition energy calculations, which correspond to the 138 MM-GBSA free energy between a ligand and individual 139 residues on UPAR, provided deeper insight into the individual 140 contributions of each amino acid to the protein-protein and 141 protein-compound interactions. Finally, we compare the 142 dynamics of the uPAR $\cdot \mathrm{uPA}$ interaction with the dynamics of 143 UPAR in complex with small molecules.

MATERIALS AND METHODS

Protein Expression and Purification. suPAR was 146 obtained by a one-step purification process as previously 147 described. $^{31}$

Crystallization and Structure Determination of UPAR 149 in Complex with Its Inhibitor, 3 (IPR-737), and 12 (IPR- 150 1175). A stabilized form of human soluble uPAR (H47C/ 151 N259C) (denoted suPARcc) was used to facilitate crystal- 152 lization of suPAR in complex with the inhibitor. suPARcc was 153 expressed and purified as previously described ${ }^{32}$ and con- 154 centrated to $16 \mathrm{mg} \mathrm{mL}^{-1}$. Crystals were formed using $1.96 \mathrm{M} 155$ ammonium sulfate, $100 \mathrm{mM}$ HEPES ( $\mathrm{pH} 7.5)$, and 2\% (w/v) 156 polyethylene glycol 400 as the precipitant solution by the 157 sitting-drop vapor diffusion method. Crystals were soaked into 158 40\% PEG4000 and $100 \mathrm{mM}$ HEPES (pH 7.5) with $1 \mathrm{mM} 3159$ (IPR-737) or 12 (IPR-1175) for $\sim 2$ days. X-ray diffraction data 160 was collected under cryogenic conditions at $100 \mathrm{~K}$. Crystals 161 were collected on beamline BL17U at the Shanghai 162 Synchrotron Radiation Facility (SSRF) and were processed, 163 integrated, and scaled together with HKL2000. ${ }^{33}$ The crystal 164 structures of the suPARcc.3 (IPR-737) complex and suPARcc. 165 12 (IPR-1175) complex were determined by the molecular 166 
Table 1. Synthesized Derivatives

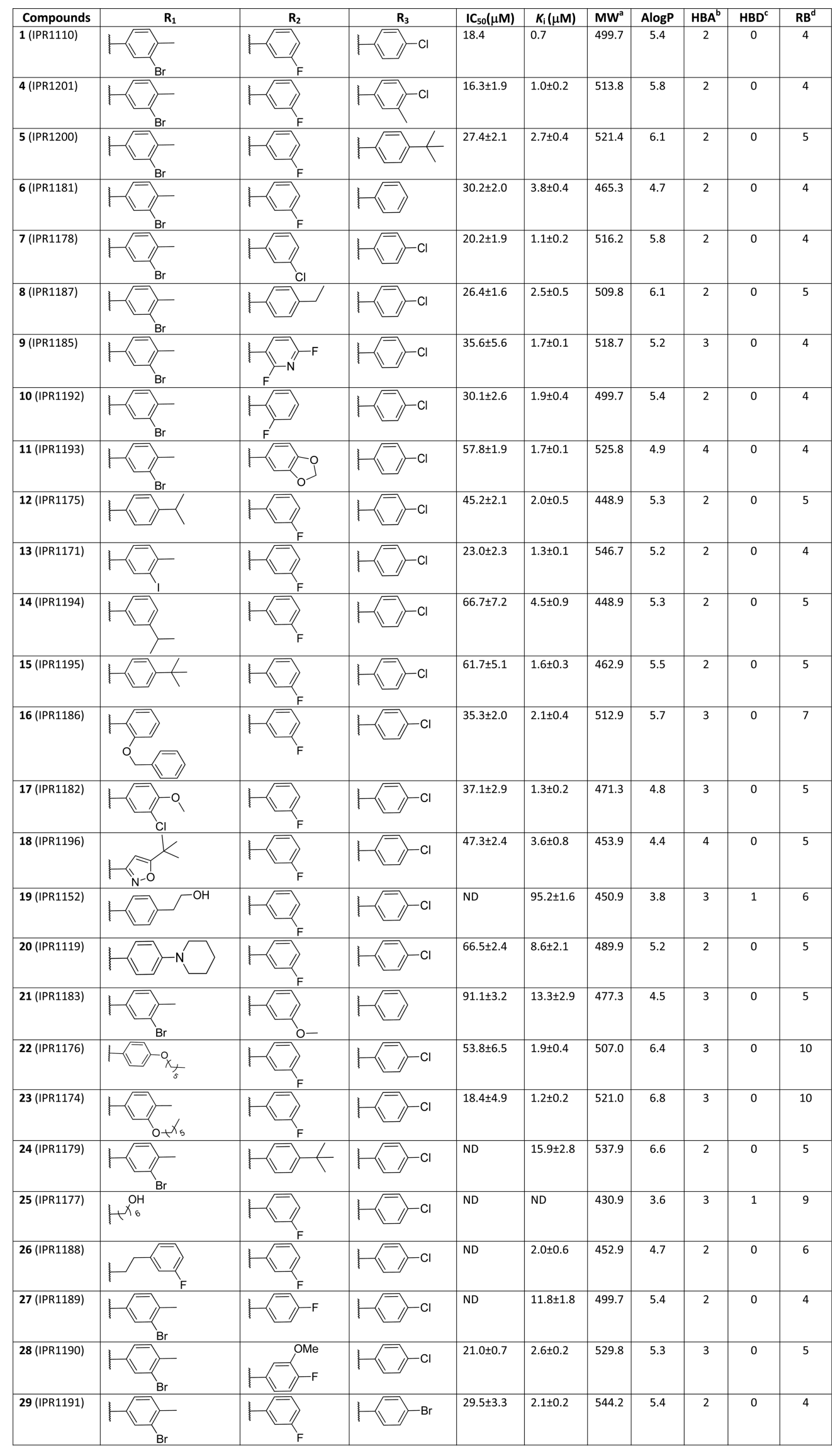


Table 1. continued

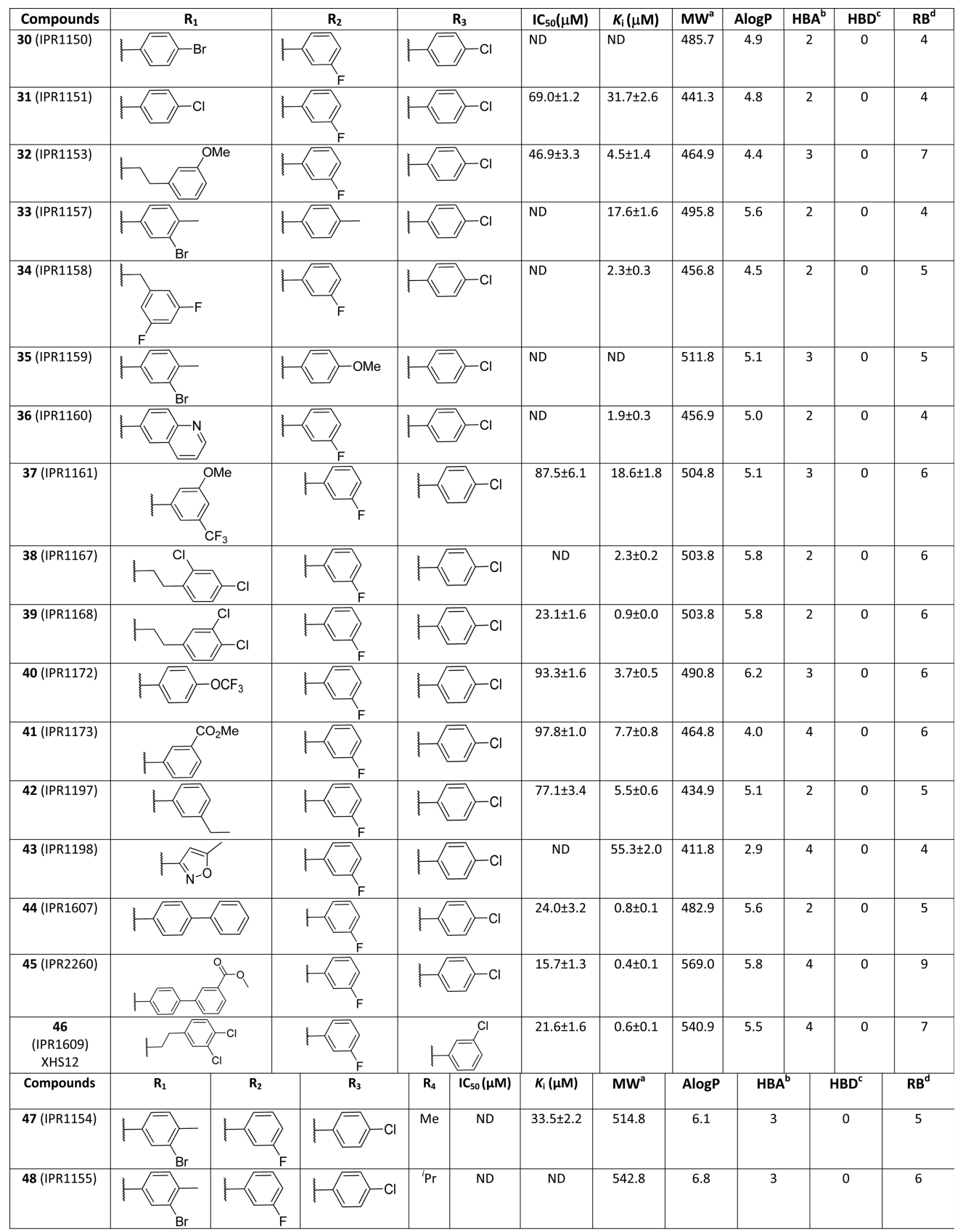

${ }^{a}$ Molecular weight. ${ }^{b}$ Hydrogen bond acceptor. ${ }^{c}$ Hydrogen bond donor. ${ }^{d}$ Rotatable bonds.

167 replacement program MOLREP ${ }^{34}$ of the CCP4 package using 168 Protein Data Bank (PDB) entry 3U74 as a search model. After 169 molecular replacement, inhibitor 3 (IPR-737) or 12 (IPR$1701175)$ was built into the model on the basis of a $2 F_{\text {obs }}-F_{\text {calc }} \sigma$ 171 weighted composite omit map, and then iterative manual model 172 building and model refinement were performed with COOT $^{35}$ 173 and REFMAC, yielding a final $R$ value of 0.2119 and an $R_{\text {free }}$ 174 value of 0.2576 in the suPARcc.3 (IPR-737) complex (Table 175 S2), while the values were 0.2349 and 0.2613 , respectively, in 176 the suPARcc-12 (IPR-1175) complex (Table S1). These 177 structures were analyzed by PyMOL. 4

178 Fluorescence Polarization. Polarized fluorescence inten179 sities were measured using an EnVision Multilabel plate reader 180 (PerkinElmer) with excitation and emission wavelengths of 485 181 and $530 \mathrm{~nm}$, respectively. ${ }^{24}$ A Thermo Scientific Nunc 384-well black microplate was used to prepare samples with a final 182 volume of $50 \mu \mathrm{L}$ in duplicate. First, the compounds were 183 serially diluted in dimethyl sulfoxide (DMSO) and further 184 diluted in $1 \times$ PBS buffer with $0.01 \%$ Triton X-100 to yield a 185 final concentration from 100 to $0.046 \mu \mathrm{M}$. Triton X-100 was 186 added in the buffer to avoid compound aggregation; $35 \mu \mathrm{L}$ of 187 the compound solution and $10 \mu \mathrm{L}$ of PBS containing UPAR 188 were added to the wells, and the mixture was incubated for at 189 least $15 \mathrm{~min}$ to allow the compound to bind to the protein. 190 Finally, $5 \mu \mathrm{L}$ of fluorescent AE147-FAM peptide was added for 191 a total volume of $50 \mu \mathrm{L}$ in each well, resulting in final uPAR and 192 peptide concentrations of 320 and $100 \mathrm{nM}$, respectively. The 193 final DMSO concentration was 2\%, which had no effect on the 194 binding of the peptide. Controls included wells containing only 195 the peptide and wells containing both protein and peptide each 196 
197 in quadruplicate to ensure the validity of the reaction assay. A 198 unit of millipolarization $(\mathrm{mP})$ was used to calculate the percent 199 inhibition of the compounds. Inhibition constants were 200 determined using the $K_{\mathrm{i}}$ calculator available at http://sw16. $201 \mathrm{im} . \mathrm{med}$.umich.edu/software/calc ki/.

202 Microtiter-Based ELISA for uPAR·uPA. High-binding 203 microplates (Greiner Bio-One) were incubated for $2 \mathrm{~h}$ at $4{ }^{\circ} \mathrm{C}$ 204 with $100 \mu \mathrm{L}$ of $2 \mu \mathrm{g} \mathrm{mL}^{-1} \mathrm{uPA}_{\mathrm{ATF}}$ in PBS. Each plate was 205 washed with $0.05 \%$ Tween 20 in PBS buffer between each step. 206 A 1:1 mixture of Superblock buffer in PBS (Thermo Fisher 207 Scientific, Inc., Waltham, MA) with $0.04 \mathrm{M} \mathrm{NaH}_{2} \mathrm{PO}_{4}$ and 0.3 $208 \mathrm{M} \mathrm{NaCl}$ buffer was used for blocking at room temperature for 1 $209 \mathrm{~h} ; 100 \mu \mathrm{L}$ of $75 \mathrm{nM}$ uPAR in PBS with $0.025 \%$ Triton X-100 210 was added with the indicated concentrations of compounds. 211 Compounds were screened initially at $50 \mu \mathrm{M}$. For concen212 tration-dependent studies, a range of compound concentrations 213 from 100 to $0.4 \mu \mathrm{M}$ were used. The final DMSO concentration 214 was $1 \%$. Following incubation for $30 \mathrm{~min}$ and subsequent 215 washing steps, the human UPAR biotinylated antibody (1:3000 216 dilution of $0.2 \mathrm{mg} \mathrm{mL}^{-1}$ BAF807, R\&D Systems, Minneapolis, $217 \mathrm{MN}$ ) in PBS containing 1\% BSA was added to the wells (100 $218 \mu \mathrm{L} /$ well) and incubated for $1 \mathrm{~h}$ to allow for the detection of 219 bound uPAR. Following washing, $100 \mu \mathrm{L}$ of streptavidin-bound 220 horseradish peroxidase ( $84 \mathrm{ng} \mathrm{mL}^{-1}$ ) in PBS containing $1 \%$ 221 BSA was added for $20 \mathrm{~min}$. The signal obtained in the presence 222 of TMB in phosphate-citrate buffer ( $\mathrm{pH} \mathrm{5)}$ and hydrogen 223 peroxide was stopped by adding a $\mathrm{H}_{2} \mathrm{SO}_{4}$ solution and detected 224 using a SpectraMax M5e instrument (Molecular Devices, 225 Sunnyvale, CA).

226 Molecular Docking. Small molecules were prepared for 227 molecular docking using Maestro (version 9.4, Schrödinger, 228 LLC, New York, NY). Compounds were first processed with 229 LigPrep (version 2.6, Schrödinger, LLC). The receptor protein 230 structure (PDB entry 3BT1 and crystal structures of the uPAR. 2313 and uPAR 12 complexes) was prepared using the Protein 232 Preparation Wizard workflow in Maestro. Bond orders were 233 assigned, hydrogen atoms added, and disulfide bonds created. 234 For the 3BT1 structure, vitronectin (chain B) was removed and 235 the missing loop at residues Arg- 83 and Ala- 84 introduced 236 using the Prime ${ }^{36}$ module in Schrödinger. The missing loops 237 from Ser-81 to Ser-90 (uPAR·3) and from Ile-129 to Lys-139 238 (UPAR.3 and UPAR.12) were reconstructed using the loop239 model class in MODELER (version 9.13). ${ }^{37}$ Five initial models 240 were constructed and refined using the "fast" molecular 241 dynamics (MD) annealing function. Five additional loop 242 models were constructed for each initial model and similarly 243 refined. A loop model was visually selected from the 25 loop 244 models that best matched the existing loop in the 3BT1 245 structure. Finally, structures were protonated at $\mathrm{pH} 7.0$ using 246 PROPKA. ${ }^{38}$

247 The binding poses of select derivatives of 1 (IPR-1110) were 248 generated using the cocrystal structure of uPAR in complex 249 with 3 (IPR-1175) as a guide in Maestro. The derivatives were 250 docked in a $21 \AA$ box centered on the complexed ligand using 251 Glide $^{39}$ (Schrödinger, LLC) in standard precision (SP) mode. 252 The common core structure of the analogues found in Table 1 253 was used to restrict the binding poses of the derivative 254 compounds. All other parameters were set to default values.

255 Molecular Dynamics Simulations. The binding poses 256 were used to run MD simulations using the AMBER14 and 257 AmberTools 15 packages. ${ }^{40}$ Each compound was assigned AM1$258 \mathrm{BCC}^{41}$ charges and gaff ${ }^{42}$ atom types using the antechamber 259 program. ${ }^{43}$ Complexes were immersed in a box of TIP3P ${ }^{44}$ water molecules. No atom on the complex was within $14 \AA$ of 260 any side of the box. The solvated box was further neutralized 261 with $\mathrm{Na}^{+}$or $\mathrm{Cl}^{-}$counterions using the tleap program.

Simulations were performed using the GPU accelerated 263 version of the pmemd program with $\mathrm{ff} 12 \mathrm{SB}^{45}$ and gaff ${ }^{42}$ force 264 fields under periodic boundary conditions. All bonds involving 265 hydrogen atoms were constrained by using the SHAKE 266 algorithm, ${ }^{46}$ and a 2 fs time step was used in the simulation. 267 The particle mesh Ewald ${ }^{47}$ (PME) method was used to treat 268 long-range electrostatics. Simulations were run at $298 \mathrm{~K}$ under 269 $1 \mathrm{~atm}$ in the NPT ensemble employing a Langevin thermostat 270 and a Berendsen barostat. Water molecules were first energy- 271 minimized and equilibrated by running a short simulation with 272 the complex fixed using Cartesian restraints. This was followed 273 by a series of energy minimizations in which the Cartesian 274 restraints were gradually relaxed from 500 to $0 \mathrm{kcal} \AA^{-2}$, and the 275 system was subsequently gradually heated to $298 \mathrm{~K}$ via a 48 ps 276 MD run. Via assignment of different initial velocities, 10277 independent simulations that are $10 \mathrm{~ns}$ in length each were 278 performed for the protein-compound structures.

279

Free Energy Calculations. In each of the 10 trajectories 280 (10 ns in length), the first 2 ns was discarded for equilibration. 281 MD snapshots were saved every 1 ps, yielding 8000 structures 282 per trajectory. A total of 80000 snapshots were generated per 283 $100 \mathrm{~ns}$ of simulation; 1000 snapshots were selected at regular 284 intervals from the 80000 snapshots for free energy calculations 285 using the cpptraj program. ${ }^{48}$ The molecular mechanics- 286 generalized Born surface area (MM-GBSA) ${ }^{49}$ method was 287 used to calculate the free energy using the MMPBSA.py script ${ }^{50} 288$ and Onufriev's GB model. ${ }^{51,52}$ Solvent-accessible surface area 289 (SASA) calculations were switched to the ICOSA method, 290 where surface areas are computed by recursively approximating 291 a sphere around an atom, starting from an icosahedron. Salt 292 concentrations were set to $0.1 \mathrm{M}$. The entropy was determined 293 by normal mode calculations ${ }^{53}$ with the nmode module from 294 100 of the 1000 snapshots used in the free energy calculations. 295 The maximal number of cycles of minimization was set to 296 10000. The convergence criterion for the energy gradient to 297 stop minimization was 0.5 .

The MM-GBSA binding free energy is expressed as

$$
\Delta G_{\mathrm{MM}-\mathrm{GBSA}}=\Delta E_{\mathrm{GBTOT}}-T \Delta S_{\mathrm{NM}}
$$

where $\Delta E_{\mathrm{GBTO}}$ is the combined internal and solvation 300 energies, $T$ is the temperature, and $\Delta S_{\mathrm{NM}}$ is the entropy 301 determined by normal mode calculation. The solvation energy 302 is determined using generalized Born (GB) solvation models 303 $\left(\Delta E_{\mathrm{GBSOL}}\right)(\mathrm{igb}=2)$ :

$$
\Delta E_{\mathrm{GBTOT}}=\Delta E_{\mathrm{GBSOLV}}+\Delta E_{\mathrm{GAS}}
$$

where $\Delta E_{\mathrm{GBSOLV}}$ is the solvation free energy and $\Delta E_{\mathrm{GAS}}$ is the 305 molecular mechanical energies (gas phase). The gas-phase 306 energies are composed of two components:

$$
\Delta E_{\mathrm{GAS}}=\Delta E_{\mathrm{ELE}}+\Delta E_{\mathrm{VDW}}
$$

where $\Delta E_{\mathrm{ELE}}$ is the nonpolar electrostatic energy and $\Delta E_{\mathrm{VDW}}$ is 308 the polar van der Waals energy. The GB solvation free energy is 309 expressed by

$$
\Delta E_{\mathrm{GBSOLV}}=\Delta E_{\mathrm{SURF}}+\Delta E_{\mathrm{GB}}
$$

where $\Delta E_{\mathrm{SURF}}$ and $\Delta E_{\mathrm{GB}}$ are the nonpolar and polar 311 contributions to the solvation free energy, respectively. All 312 the binding energies are determined by 


$$
\Delta E=E^{\mathrm{COM}}-E^{\mathrm{REC}}-E^{\mathrm{LIG}}
$$

314 where $E^{\mathrm{COM}}, E^{\mathrm{REC}}$, and $E^{\mathrm{LIG}}$ are total energies corresponding to 315 the complex, receptor, and ligand, respectively.

316 Decomposition Energy. The decomposition energy used 317 in this work was determined using the MMPBSA.py script ${ }^{50}$ 318 available in AMBER14. The script provides several schemes for 319 decomposing calculated free energies into specific residue 320 contributions using either GB or PB implicit-solvent models. 321 These schemes were developed by Gohlke and co-workers. ${ }^{54}$ 322 The per-residue decomposition scheme was used in this work. 323 Similar to the free energy calculations, we use the GB solvation 324 model from Onufriev and co-workers. ${ }^{51,52}$ The energy terms 325 are decomposed according to the scheme outlined in the 326 AMBER14 manual using the following equation:

$$
\begin{aligned}
\Delta E_{\text {decomp }}= & \sum_{j \in \text { COMPLEX } \wedge \in \text { RECEPTOR }}\left[\left\langle E^{\operatorname{COMPLEX}}(i, j)\right\rangle\right. \\
& \left.-\left\langle E^{\operatorname{RECEPTOR}}(i, j)\right\rangle\right] \\
& +\sum_{j \in \operatorname{COMPLEX} \wedge j \in \operatorname{LIGAND}}\left[\left\langle E^{\operatorname{COMPLEX}}(i, j)\right\rangle\right. \\
& \left.-\left\langle E^{\operatorname{LIGAND}}(i, j)\right\rangle\right]
\end{aligned}
$$

327 where the first and second terms represent the average 328 contribution over snapshots $i$ from the MD simulation in 329 residues $j$ on the receptor and ligand, respectively. The term $330 E(i, j)$ corresponds to the contribution of the gas-phase and 331 solvation energies, that is

$$
\begin{aligned}
E(i, j) & =E_{\mathrm{GAS}}(i, j)+E_{\mathrm{GBSOLV}}(i, j) \\
& =E_{\mathrm{VDW}}(i, j)+E_{\mathrm{ELE}}(i, j)+E_{\mathrm{GB}}(i, j)+E_{\mathrm{SURF}}(i, j)
\end{aligned}
$$

332 where $E_{\mathrm{VDW}}$ and $E_{\mathrm{ELE}}$ are the van der Waals and electrostatic 333 energies in the gas phase $\left(E_{\mathrm{GAS}}\right)$, respectively. $E_{\mathrm{GB}}$ and $E_{\mathrm{SURF}}$ are 334 the polar and nonpolar contributions to the solvation free 335 energy by the GB solvation model $\left(E_{\mathrm{GBSOLV}}\right)$, respectively. 336 Entropy is not included in the decomposition method.

337 The GB model is described in detail by Onufriev and 338 associates $^{55}$ and in the AMBER14 manual. The GB model we 339 selected (igb $=2, \mathrm{~GB}^{\mathrm{OBC}}$ model I) approximates the solvation 340 electrostatic $E_{\mathrm{GB}}$ by an analytical formula:

$$
E_{\mathrm{GB}}=-\frac{1}{2} \sum_{i j} \frac{q_{i} q_{j}}{f^{\mathrm{GB}}}\left[1-\frac{\exp \left(-\mathrm{K} f^{\mathrm{GB}}\right)}{\varepsilon}\right]
$$

341 where $r_{i j}$ is the distance between atoms $i$ and $j, R_{i}$ and $R_{j}$ are the 342 effective Born radii of atoms $i$ and $j$, respectively, $K$ is the 343 Debye-Hückel screening parameter, $\varepsilon$ is the dielectric 344 constant, and $f^{\mathrm{GB}}$ is a smooth function. Each atom in the GB 345 model is represented as a sphere with radius $\rho_{i}$ with charge $q_{i}$. 346 The $f^{\mathrm{GB}}$ function is expressed as

$$
f^{\mathrm{GB}}=\sqrt{r_{i j}^{2}+R_{i} R_{j} \exp \left(-\frac{r_{i j}^{2}}{4 R_{i} R_{j}}\right)}
$$

347 and is used to describe the distance between two atoms and 348 their effective Born radii.

349 The nonpolar contribution to the solvation free energy is 350 calculated by approximating the total SASA of the molecule:

$$
E_{\mathrm{SURF}}=\gamma \mathrm{SASA}+\beta
$$

where $\gamma$ and $\beta$ are the surface tension and offset terms, 351 respectively. The ICOSA method is used to determine 352 SASA. $^{40,54}$ In this method, surface areas are computed by 353 recursively approximating a sphere around an atom. The first 354 sphere is modeled as an icosahedron. In each subsequent step, 355 the faces of the polyhedron are divided into four equal-sized 356 triangles to better approximate the sphere.

357

Chemistry. All chemicals were purchased from commer- 358 cially available sources and used as received. Column 359 chromatography was performed with silica gel $(25-63 \mu \mathrm{m}) .360$ High-resolution mass spectra were recorded on an Agilent 6520361 Accurate Mass Q-TOF instrument. ${ }^{1} \mathrm{H}$ nuclear magnetic 362 resonance was recorded in $\mathrm{CDCl}_{3}$ or $\mathrm{DMSO}$ on a Bruker 363 $500 \mathrm{MHz}$ spectrometer. Reverse-phase liquid chromatography 364 and mass spectrometry were performed on an Agilent 1100365 LC/MSD instrument fitted with an Eclipse XBD-C18 (4.6 mm 366

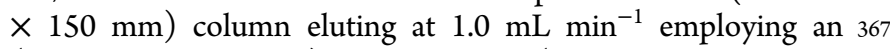
(acetonitrile/methanol)/water gradient (each containing $5 \mathrm{mM} 368$ $\mathrm{NH}_{4} \mathrm{OAc}$ ) from 70 to $100 \%$ acetonitrile/methanol over $15 \mathrm{~min} 369$ and holding at $100 \%$ acetonitrile/methanol for $2 \mathrm{~min}$. Chemical 370 shifts are reported in parts per million using either residual 371 $\mathrm{CHCl}_{3}$ or DMSO as an internal reference. All compounds are 372 $>95 \%$ pure unless otherwise stated. Syntheses of 3-(hexyloxy)- 373 aniline and 3-(hexyloxy)-4-methylaniline were performed using 374 a protocol described by Marco and co-workers. ${ }^{56} \beta-375$ Diketoesters were synthesized with modification according to 376 Milagre and co-workers. ${ }^{57}$ Derivatives of $\mathbf{1}$ were synthesized by 377 a modified procedure of Rose and co-workers. ${ }^{58}$ Full 378 compound characterization is provided in the Supporting 379 Information.

RESULTS

381

Synthesis of Pyrrolinones, Stability, Reactivity, and 382 Selectivity Studies. The ease of synthesis of 1 (IPR-1110) 383 prompted us to prepare 46 derivatives to explore the uPAR 384 uPA binding interface (Table 1). 1 (IPR-1110) was modified at 385 three sites, $R_{1}-R_{3}$. Most of the structure-activity exploration 386 was focused on $R_{1}$ and $R_{2}$. The synthesis of these compounds 387 was straightforward (Figure S1) as described previously. ${ }^{23} \quad 388$

The pyrrolinone core structure of $\mathbf{1}$ suggests that 389 nucleophilic residues within a protein could potentially form 390 covalent adducts through nucleophilic attack at the carbon 391 bearing the hydroxyl group of the core five-membered ring. To 392 explore this possibility, we employed electrospray ionization 393 (ESI) mass spectrometry. As shown in Figure S2, there was no 394 adduct formation when uPAR was incubated with the 395 compound, ruling out nonspecific covalent bond formation as 396 the mechanism by which the pyrrolinone compounds 397 antagonize UPAR-protein interactions. Next, we investigated 398 the stability of the compound in buffer (PBS), methanol, and 399 uPAR using high-performance liquid chromatography (Figure 400 S3). The UV spectra for all three conditions were identical 401 (Figure S3a), and the mass spectra corresponding to the major 402 peaks were also identical (Figure S3b). This suggests that the 403 compound is stable both in buffer and in the presence of uPAR. 404

Finally, we investigated the selectivity of the pyrrolinone 405 compounds by testing them for inhibition of an unrelated 406 protein-protein interaction between the $\alpha$ and $\beta$ subunits of 407 the CaV2.2 calcium channel (Figure S4). The $\mathrm{CaV} \alpha \beta 408$ interaction is mediated by a tight single-digit nanomolar 409 interaction that occurs over a large interface consisting 410 primarily of an $\alpha$ helix of the $\alpha$ subunit binding to a large 411 well-defined pocket on the $\beta$ subunit (Figure S4). Compound 1412 

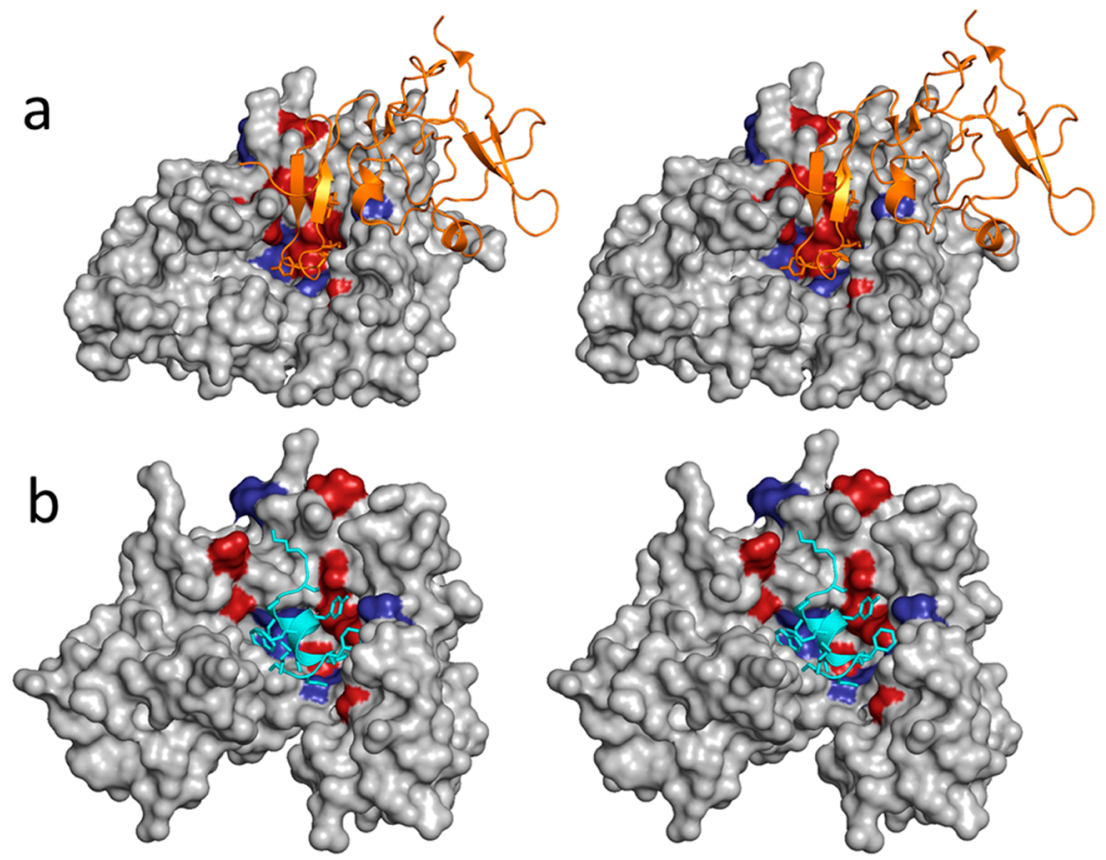

Figure 1. (a) Three-dimensional structure of the uPAR $u \mathrm{uPA}_{\mathrm{ATF}}$ complex (PDB entry 2FD6). uPAR is shown as a gray solvent-accessible surface. Residues whose mutations to alanine result in changes in binding affinity of $\geq 1 \mathrm{kcal} \mathrm{mol}^{-1}$ are colored red, while those that lead to changes between 0.5 and $1 \mathrm{kcal} \mathrm{mol}^{-1}$ are colored purple. $\mathrm{uPA}_{\mathrm{ATF}}$ is shown as an orange cartoon. (b) Three-dimensional structure of uPAR in complex with the AE157 peptide (PDB entry 1YWH). The peptide is shown as a cyan cartoon, and side chains that come in contact with uPAR are shown as capped sticks. UPAR is rendered and color-coded in a manner similar to that of panel a.

413 (Figure S4) showed no inhibition of this interaction up to 25 $414 \mu \mathrm{M}$. The effect of 1 on the $\operatorname{CaV} \alpha \beta$ interaction at 50 and 100 $415 \mu \mathrm{M}$ can be attributed to aggregation considering the sharp rise 416 in activity from nearly $0 \%$ inhibition at $25 \mu \mathrm{M}$ to more than $41775 \%$ inhibition at $50 \mu \mathrm{M}$. This was not observed for uPAR, 418 whereby 1 exhibited a gradual increase in its level of inhibition 419 of binding of AE-147 to uPAR (Figure S4). These studies 420 confirm that the compounds bind to UPAR in a selective 421 manner as evidenced by the lack of activity against another 422 unrelated protein-protein interaction. It is possible that 423 compound 1 inhibits interactions that are similar to the $424 \mathrm{uPAR} \cdot \mathrm{uPA}$ interaction. Future studies that explore compounds 425 in cell culture should explore these compounds for inhibition of 426 interactions that are similar to uPAR.uPA interactions.

427 Crystal Structures of Compounds Bound to uPAR. The 428 crystal structure of UPAR was previously determined in 429 complex with ATF (PDB entry 2FD6) (Figure 1a) or $\alpha$-helical 430 peptide AE-147 (PDB entry 1YWH) (Figure 1b). These 431 structures revealed a large interface between UPAR and UPA 432 that contains several hot spots, including Leu-55, Tyr-57, Leu433 66, Asp-140, and Leu-150. All the hot spots on uPAR at the $434 \mathrm{uPAR} \cdot \mathrm{uPA}$ interaction are listed in Table S1. We hypothesized 435 that compounds that disrupt the protein-protein interaction 436 between uPAR and uPA likely directly engage these hot spots. 437 To test this hypothesis, we resorted to X-ray crystallography to 438 determine the structure of $\mathbf{1}$ (IPR-1110) and derivatives in 439 complex with uPAR. We also attempted to determine the 440 structures of pyrazole, piperidinone, pyrrolidinone, and butan441 amine compounds that we had previously shown to bind to $442 \mathrm{uPAR}^{21}$ We obtained structures for two compounds bound to 443 uPAR, namely, pyrrolinone 12 (IPR-1175) (Figure 2 and Table 444 S2) and pyrazole 3 (IPR-737) (Figure 3 and Table S3).

445 The structure of uPAR in complex with 12 reveals that the 446 compound is ensconced deeply in the pocket that is occupied by the growth factor-like domain of uPA in its complex with 447 uPAR (Figure 2a). The $R_{1}$ isopropylphenyl group of the 448 compound points toward the interior of the hydrophobic 449 pocket of UPAR. This pocket accommodates the residues 450 located on the loop of the $\beta$ turn of the GFD domain of uPA. 451 The substituent makes direct contacts with Leu-150, Leu-168, 452 Val-125, Leu-55, and Arg-53 (Figure 2b). Most of these 453 interactions are hydrophobic except for the cation $-\pi 454$ interaction with Arg-53. Cation $-\pi$ interactions are commonly 455 observed in protein-compound structures and can contribute 456 up to $1 \mathrm{kcal} \mathrm{mol}^{-1}$ to the free energy of binding. ${ }^{59,60}$ Here, the 457 benzene ring of the $R_{1}$ group of $\mathbf{1 2}$ (IPR-1175) directly faces 458 one of the $\mathrm{N} \omega$ atoms of the guanidinium ion of Arg-53. The 459 $\mathrm{N} \omega$ atom is $2.5 \AA$ from one of the ortho carbon atoms of the 460 benzene ring. Unlike $R_{1}$, the chlorobenzene $R_{3}$ group of 12461 (IPR-1175) points in the opposite direction and occupies a 462 pocket surrounded by residues Thr-27, Val-29, Arg-142, and 463 Leu-40 on uPAR. The $R_{3}$ group is more exposed to solvent 464 than $R_{1}$ and does not occupy a pocket composed entirely of 465 hydrophobic residues as evidenced by the presence of Arg-142. 466 Finally, the $\mathrm{R}_{2}$ group of $\mathbf{1 2}$ does not occupy a well-defined 467 pocket on UPAR, but it is worth noting that this group is also 468 involved in a cation $-\pi$ interaction with Arg-53 of uPAR (Figure 469 $2 \mathrm{~b})$. Interestingly, the $\mathrm{R}_{1}$ and $\mathrm{R}_{3}$ groups of $\mathbf{1 2}$ occupy the same 470 position as uPA hot spots Ile-28 and $\mathrm{Tyr}-24$, respectively 471 (Figure 2c).

472

3 (IPR-737) adopts a binding mode different from that of 12473 (IPR-1175) (Figure 3). 3 (IPR-737) binds outside the 474 hydrophobic pocket of UPAR that is occupied by side chains 475 of the $\beta$-turn loop of the GFD domain of uPA (Figure 3a). 476 Unlike 12 (IPR-1175), the compound engages fewer hot spots 477 on uPAR (Figure 3b). Only one of the uPAR hot-spot residues 478 (Leu-66) comes in contact with 3 (IPR-737). Interestingly, 479 analysis of the crystal structure of UPAR in complex with the 480 


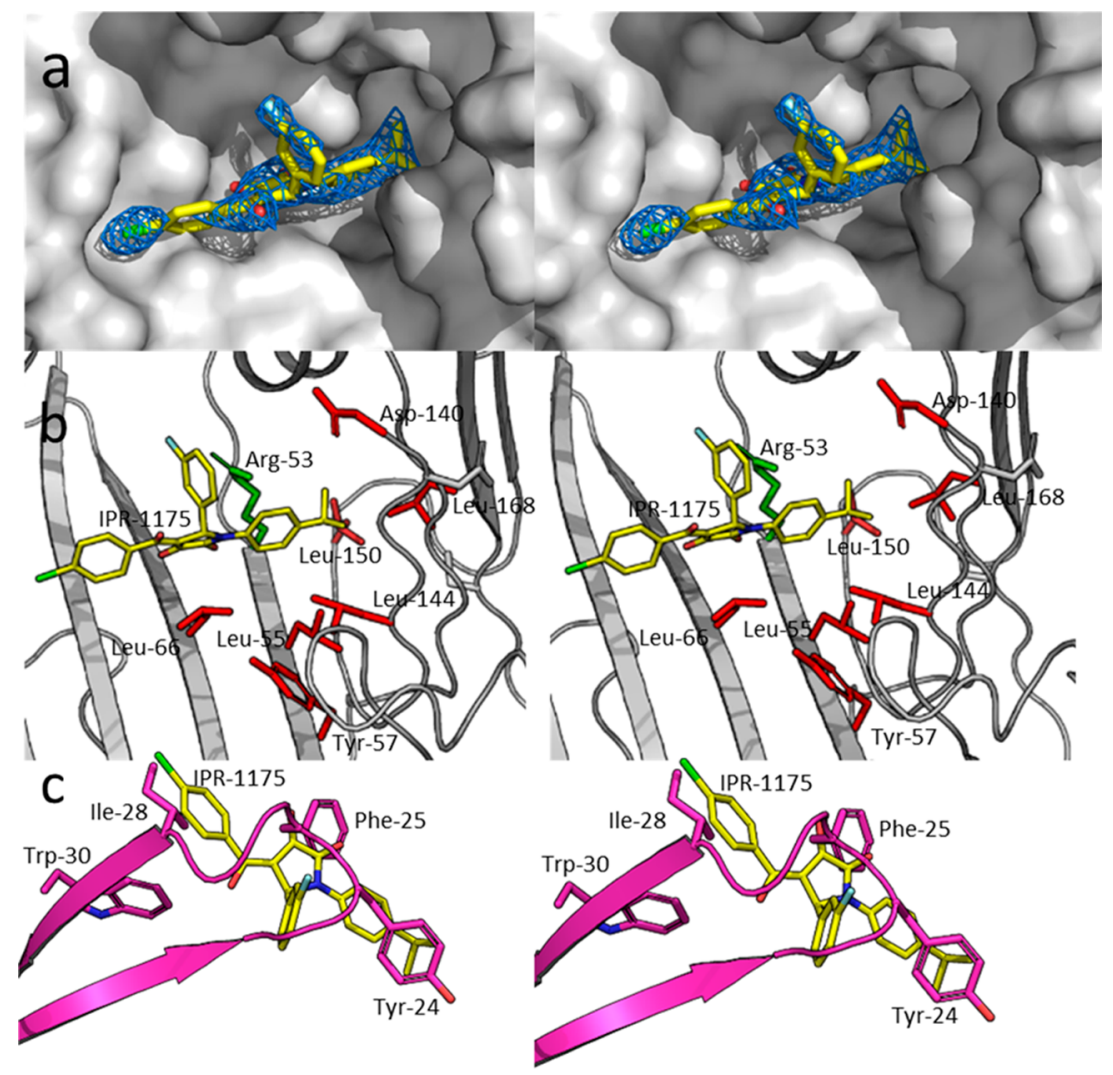

Figure 2. Mode of binding of 12 (IPR-1175) upon the central ligand binding cavity of suPARcc. suPARcc is shown as a gray solvent-accessible surface. 12 (IPR-1175) is shown as capped sticks. Atoms are color-coded by atom type with $\mathrm{N}, \mathrm{C}, \mathrm{O}, \mathrm{Cl}$, and $\mathrm{F}$ colored blue, yellow, red, green, and light blue, respectively. The $2 F_{\mathrm{o}}-F_{\mathrm{c}}$ electron density map of 12 (IPR-1175) at $1 \sigma$ is colored blue. (b) Crystal structure of $\mathbf{1 2}$ (IPR-1175) bound to uPAR except that UPAR is shown as capped sticks to highly hot-spot residues located within the uPAR cavity. Hot spots are shown as red capped sticks. Arg-53, which is not considered a hot spot, is colored green. (c) Crystal structure of the uPAR-12 (IPR-1175) complex superimposed on the

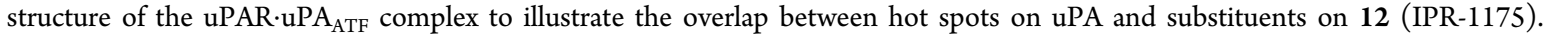

481 AE-147 peptide shows that two hydrophobic residues on the 482 peptide (Phe-5 and Tyr-8) come in direct contact with Ile-66 of 483 uPAR (Figure 1b). Hence, interaction with Ile-66 may explain 484 why 3 (IPR-737) can disrupt binding of AE-147 to uPAR. 485 Engagement of Ile-66, however, is not sufficient to disrupt the 486 full uPAR-uPA $A_{\text {ATF }}$ interaction. Ile-66 of 3 (IPR-737) binds to $487 \mathrm{UPAR}$ at a site that is occupied by Ile- 28 and Trp-30 of uPA. 488 These two residues are located on the $\beta$ strands of the $\beta$ hairpin 489 of the GFD domain of uPA (Figure 3c). This is in contrast to 49012 (IPR-1175), which overlaps with amino acids located on the 491 loop region of the $\beta$ hairpin (Figure 2c).

492 Structure-Activity Relationship. We prepared 46 493 derivatives of 1 (IPR-1110) to explore the uPAR binding site 494 at the uPAR $u P A$ interface. Substituents at $R_{1}-R_{3}$ of 1 (IPR495 1110) were explored. The binding mode of 12 (IPR-1175) 496 (Figure 2a,b) shows that $\mathrm{R}_{1}$ groups point toward a large 497 hydrophobic cavity occupied by several uPA hot-spot residues. 498 An aromatic group at $R_{1}$ was generally required for inhibition of $499 \mathrm{uPAR} \cdot \mathrm{uPA}$ interaction. This is evidenced by complete loss of 500 activity of 25 (IPR-1177), which lacks an aromatic ring at $\mathrm{R}_{1}$. 501 There are six compounds without an aromatic ring directly 502 attached to the central pyrrolidone ring: $26,32,34,38,39$, and 503 46; in these compounds, a methylene (34) or an ethylene (26, $50432,38,39$, and 46) group separates the central pyrrolinone ring 505 with a benzene ring. Except for 26, 34, and 38, the compounds 506 retained the ability to inhibit the UPAR.uPA protein-protein 507 interaction with $\mathrm{IC}_{50}$ values similar to that of 1 (IPR-1110) for 50839 and 46. All three compounds that did not inhibit uPAR.uPA interaction have fluorine atoms on the aromatic ring at $\mathrm{R}_{1}, 509$ suggesting that highly polar groups may not be suitable for the 510 binding site on uPAR. Interestingly, the compounds bind to 511 uPAR with a $K_{\mathrm{i}}$ of $1-2 \mu \mathrm{M}$. Replacing the bromine atom at $\mathrm{R}_{1} 512$ with an iodine such as in $\mathbf{1 3}$ (IPR-1171) had no effect on the 513 $\mathrm{IC}_{50}$ but gave a 2 -fold reduction in binding affinity. Removal of 514 the bromine group at the meta position and replacing the 515 methyl group with an isopropoxy group in $\mathbf{1 2}$ (IPR-1175) or a 516 tert-butyl group such as in 15 (IPR-1195) led to a 2-3-fold 517 increase in the $\mathrm{IC}_{50}$ and a nearly 2 -fold increase in $K_{\mathrm{i}}$. A 518 benzyloxy group in 16 (IPR-1186) increased the $\mathrm{IC}_{50}$ and $K_{\mathrm{i}}$ by 519 3 -fold versus those of 1 (IPR-1110). Generally, replacing the 520 hydrogen atoms at the meta position of the aromatic ring 521 directly attached to the central pyrrolidone ring reduced activity 522 substantially as evidenced by a 5 -fold increase in $\mathrm{IC}_{50}$ and a 20- 523 fold increase in $K_{\mathrm{i}}$ for 37 . Larger groups at one of the meta 524 positions were also generally undesirable as most compounds 525 had pronounced increases in $\mathrm{IC}_{50}$ such as $\mathbf{3 6}$ and $\mathbf{4 1}$. For these 526 compounds, it is interesting that 36 retained almost all of its 527 binding affinity as evidenced by a $K_{\mathrm{i}}$ of $1.9 \mu \mathrm{M}$ that was 2 -fold 528 higher than that of 1 (IPR-1110). Compound 41 had reduced 529 affinity, but its $K_{\mathrm{i}}$ value was within $10 \mu \mathrm{M}$. Introduction of 530 various substituents at the para position generally weakened the 531 ability to disrupt the UPAR.uPA protein-protein interaction 532 (e.g., 19 and 36), except for 44 (IPR-1607) and 45 (IPR-2260). 533 Both of these compounds had $\mathrm{IC}_{50}$ values similar to that of $\mathbf{1} 534$ (IPR-1110). Interestingly, the compound showed a nearly 2- 535 


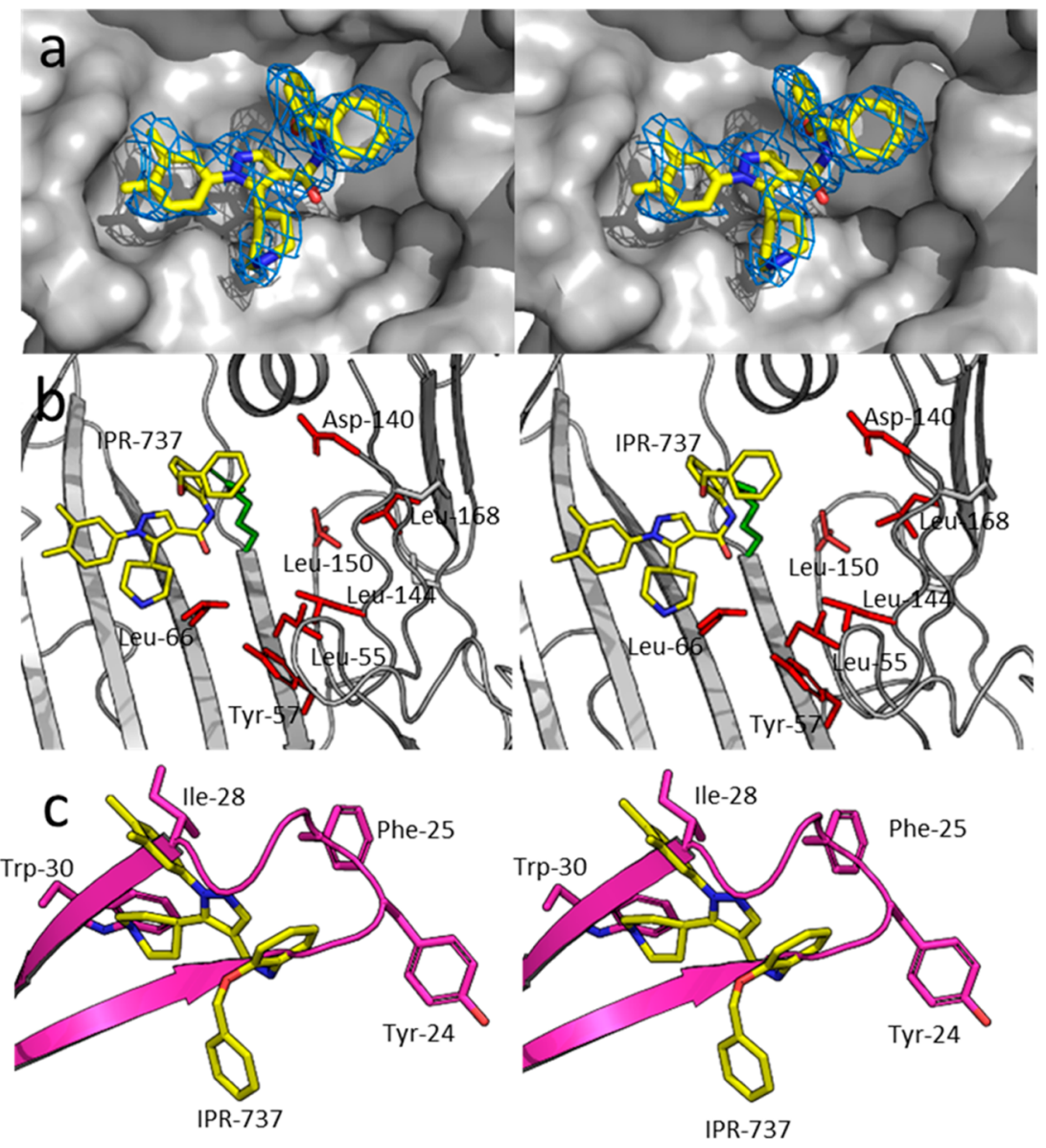

Figure 3. Mode of binding of 3 (IPR-737) upon the central ligand binding cavity of suPARcc. suPARcc is shown as a gray solvent-accessible surface. 3 (IPR-737) is shown as capped sticks. Atoms are color-coded by atom type with $\mathrm{N}, \mathrm{C}, \mathrm{O}, \mathrm{Cl}$, and $\mathrm{F}$ colored blue, yellow, red, green, and light blue, respectively. A $2 F_{\mathrm{o}}-F_{\mathrm{c}}$ electron density map of 3 (IPR-737) at $1 \sigma$ is colored blue. (b) Crystal structure of 3 (IPR-737) bound to uPAR except that UPAR is shown as capped sticks to highly hot-spot residues located within the uPAR cavity. Hot spots are shown as red capped sticks. (c) Crystal structure of the uPAR·3 (IPR-737) complex superimposed on the structure of the uPAR.uPA $\mathrm{ATF}_{\mathrm{A}}$ complex to illustrate the overlap between hot spots on uPA and substituents on 3 (IPR-737).

536 fold increase in its binding affinity for UPAR as evidenced by a $537 K_{\mathrm{i}}$ of $0.4 \mu \mathrm{M}$ compared with a $K_{\mathrm{i}}$ of $0.9 \mu \mathrm{M}$ for 1 (IPR-1110). 538 Despite the highly favorable cation $-\pi$ interaction of the $R_{2}$ 539 group with Arg-53, the substituent has significant exposure to 540 solvent. The addition of a methoxy group at the meta position 541 of the aromatic ring of $\mathrm{R}_{2}(\mathbf{2 1}$ and $\mathbf{2 8})$ reduced the binding 542 affinity and weakened the potency for inhibition of uPAR $u$ uPA 543 interaction. Replacing the fluorine on 4 (IPR-1201) with a 544 chlorine on 7 (IPR-1178), however, does not have much 545 impact on the binding affinity or inhibition of uPAR.uPA. 546 Moving the fluorine from the meta to ortho position on 10 547 reduces the $K_{\mathrm{i}}$ and $\mathrm{IC}_{50}$ by 2 -fold. It is interesting to note that a 548 methoxy group at the meta position of 21 has a much more 549 significant impact on both binding with a 10 -fold reduction in $550 K_{\mathrm{i}}$ and inhibition of the protein-protein interaction as 551 evidenced by a nearly 5-fold increase in $\mathrm{IC}_{50}$. Replacing the 552 hydrogen atom with a fluorine atom of this compound to 553 generate 28 improves the $K_{\mathrm{i}}$ and $\mathrm{IC}_{50}$ by 5 -fold versus those of 554 compound 21 . In compound 24 , we introduced a tert-butyl 555 group at the para position of the aromatic ring at $\mathrm{R}_{2}$, which led 556 to complete abrogation of the inhibition of uPAR $\cdot \mathrm{uPA}$ 557 interaction $\left(\mathrm{IC}_{50}\right.$ not determined), although direct binding to 558 uPAR was still detected but its level was reduced by more than 559 an order of magnitude $\left(K_{\mathrm{i}}=15.9 \pm 2.8 \mu \mathrm{M}\right)$. A nearly similar 560 effect was observed for 33 (IPR-1157), and an even more dramatic effect when a methoxy group was introduced at the 561 para position of $\mathrm{R}_{2}$ with no inhibition or binding detected 562 within the $100 \mu \mathrm{M}$ range.

Molecular Dynamics Simulations and Free Energy 564 Calculations. To further explore the interaction between 565 compounds and individual amino acids at the uPAR.uPA 566 interface, we resorted to explicit-solvent molecular dynamics 567 simulations and free energy calculations. We also performed 568 free energy decomposition calculations to investigate the 569 interaction of each amino acid with the compounds. First, we 570 generated a model for the structure of 12 derivatives of 1 (IPR- 571 1110 ) bound to uPAR using the binding mode of 12 (IPR- 572 1175). These compounds were selected to ensure a uniform 573 distribution across the range of binding affinities. Along with 3574 (IPR-737) and 12 (IPR-1175), these complexes were subjected 575 to $100 \mathrm{~ns}$ explicit-solvent molecular dynamics simulations. 576 Snapshots were collected at regular intervals, and free energy 577 calculations using the MM-GBSA approach were conducted for 578 each of the compounds as shown in Figure $4 \mathrm{a}$ and Table S4. It $579 \mathrm{f} 4$ is worth noting that end-point free energy calculations cannot 580 accurately reproduce the absolute value of the free energy of 581 binding. These calculations, however, have been shown in 582 numerous studies, including ours, to accurately rank-order 583 protein-compound complexes. The MM-GBSA free energy 584 consists of five components, namely, the nonpolar and polar 585 

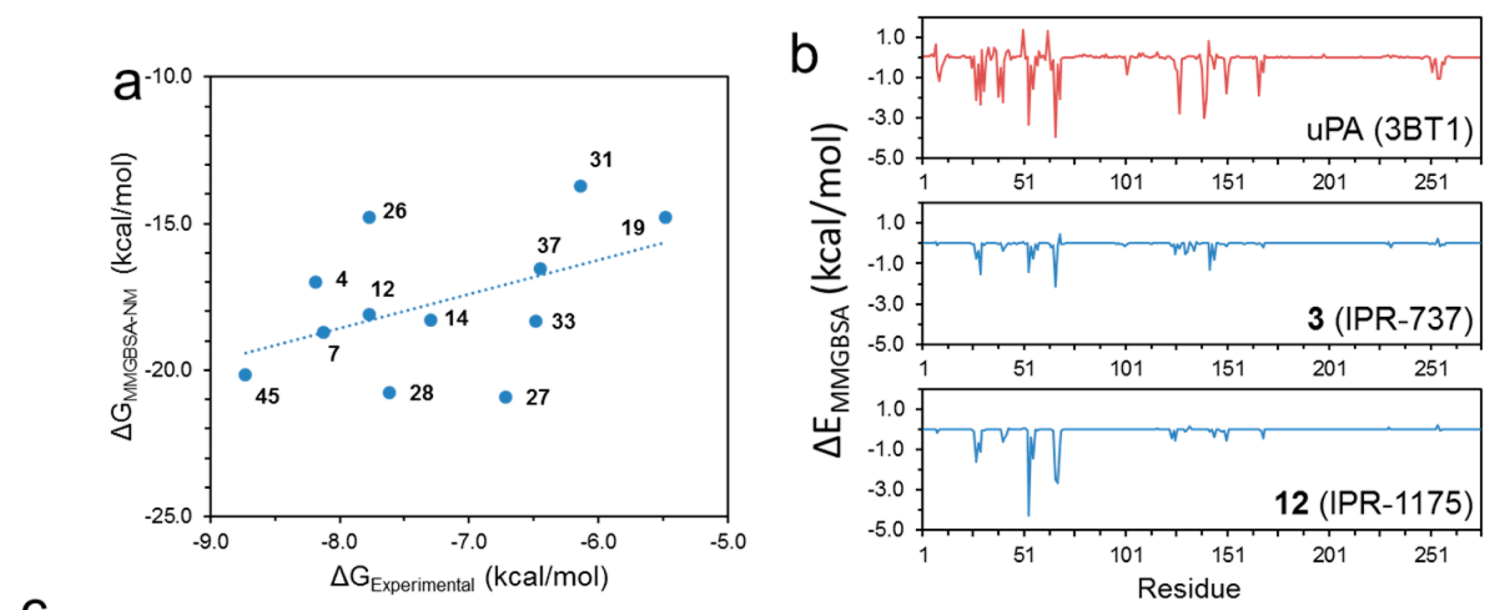

C

\begin{tabular}{|c|c|c|c|c|c|c|c|c|c|c|c|c|c|c|c|c|c|c|c|c|c|}
\hline Compound & $\Delta \mathrm{E}_{\mathrm{R} 25}$ & $\Delta \mathrm{E}_{\mathrm{T} 27}$ & $\Delta \mathrm{E}_{\mathrm{L40}}$ & $\Delta \mathrm{E}_{\mathrm{k} 50}$ & $\Delta \mathrm{E}_{\mathrm{T} 51}$ & $\Delta \mathrm{E}_{\text {R53 }}$ & $\Delta \mathrm{E}_{\mathrm{L55}}$ & $\Delta \mathrm{E}_{\mathrm{Y} 57}$ & $\Delta \mathrm{E}_{\mathrm{L66}}$ & $\Delta \mathrm{E}_{0102}$ & $\Delta \mathrm{E}_{5104}$ & $\Delta \mathrm{E}_{\mathrm{E} 106}$ & $\Delta \mathrm{E}_{\mathrm{V} 125}$ & $\Delta \mathrm{E}_{\mathrm{E} 127}$ & $\Delta \mathrm{E}_{0140}$ & $\Delta \mathrm{E}_{0141}$ & $\Delta \mathrm{E}_{\mathrm{H} 143}$ & $\Delta \mathrm{E}_{\mathrm{L} 144}$ & $\Delta \mathrm{E}_{\mathrm{L} 150}$ & $\Delta \mathrm{E}_{\mathrm{P} 151}$ & $\Delta \mathrm{E}_{\mathrm{L} 168}$ \\
\hline UPA (3BT1) & -0.54 & -2.14 & -2.25 & 1.40 & -0.06 & -3.33 & -1.60 & -0.43 & -3.93 & -0.22 & 0.02 & -0.01 & -0.53 & -2.79 & -2.09 & 0.80 & -0.01 & -0.58 & -1.77 & -0.48 & -0.76 \\
\hline 3 (IPR-737) & -0.05 & -0.78 & -0.38 & 0.09 & 0.00 & -1.41 & -0.74 & -0.32 & -2.12 & 0.05 & 0.00 & 0.03 & -0.52 & -0.23 & 0.05 & 0.04 & -0.01 & -0.82 & -0.12 & 0.00 & -0.22 \\
\hline 31 (IPR-1151) & -0.02 & -0.87 & -0.23 & 0.13 & 0.01 & -3.75 & -1.74 & -0.07 & -2.37 & 0.02 & 0.00 & 0.02 & -0.55 & -0.10 & 0.03 & 0.03 & -0.01 & -0.56 & -0.53 & -0.01 & -0.23 \\
\hline 19 (IPR-1152) & 0.02 & -1.47 & -0.46 & 0.08 & 0.03 & -3.53 & -1.38 & -0.07 & -2.28 & 0.02 & 0.00 & 0.02 & -0.69 & -0.08 & 0.02 & 0.03 & -0.02 & -0.42 & -0.63 & -0.01 & -0.46 \\
\hline 33 (IPR-1157) & -0.07 & -0.84 & -0.08 & 0.10 & 0.02 & -3.98 & -1.90 & -0.09 & -2.28 & 0.02 & 0.00 & 0.02 & -0.90 & -0.21 & 0.03 & 0.03 & -0.03 & -0.67 & -0.67 & -0.01 & -0.56 \\
\hline 37 (IPR-1161) & 0.01 & -1.87 & -0.60 & 0.14 & 0.03 & -3.46 & -1.46 & -0.06 & -2.64 & 0.02 & 0.00 & 0.01 & -0.63 & -0.10 & 0.03 & 0.03 & -0.02 & -0.55 & -0.47 & -0.01 & -0.23 \\
\hline 12 (IPR-1175) & 0.01 & -1.60 & -0.58 & 0.08 & 0.04 & -4.24 & -1.41 & -0.07 & -2.50 & 0.02 & 0.00 & 0.01 & -0.55 & 0.00 & 0.03 & 0.02 & -0.01 & -0.39 & -0.53 & -0.01 & -0.40 \\
\hline 7 (IPR-1178) & -0.07 & -0.80 & -0.13 & 0.07 & 0.02 & -3.87 & -2.13 & -0.11 & -2.32 & 0.02 & 0.00 & 0.02 & -0.93 & -0.18 & 0.02 & 0.03 & -0.02 & -0.65 & -0.62 & -0.01 & -0.50 \\
\hline 26 (IPR-1188) & -0.03 & -0.93 & -0.20 & 0.14 & 0.01 & -3.83 & -1.72 & -0.16 & -2.30 & 0.01 & 0.00 & 0.02 & -0.55 & -0.12 & 0.03 & 0.03 & -0.01 & -0.47 & -0.72 & -0.01 & -0.29 \\
\hline 27 (IPR-1189) & -0.04 & -0.70 & -0.18 & 0.07 & 0.02 & -4.58 & -1.98 & -0.05 & -1.90 & 0.02 & 0.00 & 0.02 & -0.88 & -0.22 & 0.03 & 0.03 & -0.03 & -0.55 & -0.69 & -0.02 & -0.36 \\
\hline 28 (IPR-1190) & -0.04 & -0.69 & -0.15 & 0.10 & -0.01 & -3.98 & -1.84 & -0.09 & -2.16 & 0.02 & 0.00 & 0.02 & -1.47 & -0.50 & 0.04 & 0.04 & -0.15 & -1.01 & -0.69 & -0.02 & -0.57 \\
\hline 14 (IPR-1194) & -0.08 & -0.77 & -0.14 & 0.13 & 0.01 & -4.86 & -1.86 & -0.02 & -2.36 & 0.01 & 0.00 & 0.01 & -0.80 & -0.16 & 0.03 & 0.03 & -0.01 & -0.49 & -0.67 & -0.01 & -0.37 \\
\hline 4 (IPR-1201) & -0.10 & -1.01 & -0.31 & 0.11 & -0.01 & -3.81 & -1.44 & -0.19 & -2.27 & 0.03 & 0.00 & 0.02 & -0.85 & -0.19 & 0.03 & 0.03 & -0.03 & -0.73 & -0.71 & -0.01 & -0.43 \\
\hline 45 (IPR-2260) & -0.08 & -0.96 & -0.23 & 0.14 & -0.01 & -2.62 & -1.24 & -0.08 & -1.91 & 0.02 & 0.00 & 0.02 & -1.16 & -0.41 & 0.04 & 0.03 & -0.03 & -0.46 & -1.12 & -0.17 & -1.72 \\
\hline
\end{tabular}

Figure 4. (a) Plot of the MM-GBSA free energy vs inhibition constant $K_{\mathrm{i}}$ that was measured using the fluorescence polarization assay. The correlation coefficients of all 12 selected derivatives are as follows: $r=0.48, \rho=0.41$, and $\tau=0.29$. (b) Decomposition of the free energy of binding for uPA, 12 (IPR-1175), and 3 (IPR-737). The decomposition energy consists of the interaction energy between the ligand and each residue on uPAR determined for a collection of snapshots that were obtained from molecular dynamics simulations. (c) Decomposition energies for a select number of hot-spot and non-hot-spot residues for a set of 1 (IPR-1110) derivatives.

586 potential energies, the polar and nonpolar solvation energies, 587 and the entropy (Table S4). The calculated MM-GBSA free 588 energy correlated positively with the experimentally determined $589 K_{\mathrm{i}}$ values with a Pearson's $r$ of 0.8 , a Spearman's $\rho$ of 0.41 , and a 590 Kendall's $\tau$ of 0.29 (Figure 4a).

591 To gain deeper insight into the interaction of the small 592 molecules with individual residues on UPAR, a decomposition 593 energy calculation was performed for each compound (Figure $5944 \mathrm{~b}$ ). The decomposition energy consists of polar and nonpolar 595 interaction potential energy, and the polar and nonpolar 596 solvation energies. These calculations were also performed for 597 the uPAR $u$ uPA complex. On uPAR, mutations at 21 residues at 598 the uPAR.uPA interface significantly impaired the interaction. ${ }^{18}$ 599 Among these residues, only a subset is considered hot spots by 600 the traditional definition $\left(\Delta \Delta G>1 \mathrm{kcal} \mathrm{mol}^{-1}\right)$. Additionally, 601 not all hot spots are included in this list of residues. We 602 examine the local interaction between uPA and compounds 603 with $\mathrm{UPAR}$ at these residues (Figure 4c). As expected, uPA 604 strongly engaged these residues. The change in free energy 605 from the experimental alanine scan correlated with the per606 residue decomposition $(r=0.38, \rho=0.17$, and $\tau=0.01)$. When 607 only hot-spot residues are considered, the correlation decreases 608 to $r=0.18, \rho=0.11$, and $\tau=0.10$. At the 21 residues 609 mentioned above, the correlation is $r=0.11, \rho=0.28$, and $\tau=$ 610 0.19. Comparison of the decomposition energies of 3 (IPR611737 ) and 12 (IPR-1175) provides insight into the residues that contribute to the ability of $\mathbf{1 2}$ to disrupt the protein-protein 612 interaction. Compound 3 (IPR-737) decomposition interaction 613 energies were much weaker than those observed for uPA. In 614 contrast, 12 (IPR-1175) showed much more favorable 615 interaction energies that were comparable to those of uPA, 616 particularly to Thr-27, Leu-55, Leu-66, and Arg-53. It is 617 interesting to note that among all the 21 residues considered, 618 the experimental binding affinity of compounds correlated most 619 strongly with their interaction energies with Leu-150 and Leu- 620 168 with Pearson correlation coefficients of 0.57 and $0.52,621$ respectively (Table S5). In addition, the experimental kinetic 622 rate constants were also compared to the residue decom- 623 position energies of these derivatives (Table S6).

624

Arg-53 Enhances Binding through Cooperativity. Arg- 625 53 is not considered a hot spot, because alanine scanning 626 studies revealed that it contributes only $0.7 \mathrm{kcal} \mathrm{mol}^{-1},{ }^{18}$ but it 627 appears to play a critical role in the binding of small molecules 628 as we have demonstrated previously for 2. Considering the 629 cation $-\pi$ interaction of 12 with Arg-53, we hypothesized that 630 the residue also plays an essential role in the activity of the 631 compound. We explored the possibility that Arg-53 may be 632 enhancing interaction of the compound with hot spots through 633 cooperative binding. MM-GBSA free energy calculations for 634 double mutants that include Arg-53 were also conducted along 635 with separate MM-GBSA calculations for the individual 636 residues. We ran explicit-solvent molecular dynamics simu- 637 
638 lations and free energy calculations for both the single- and 639 double-mutation states of the uPAR.uPA (Figure 5a) and 640 uPAR 12 (Figure 5b) complexes (Table S7).
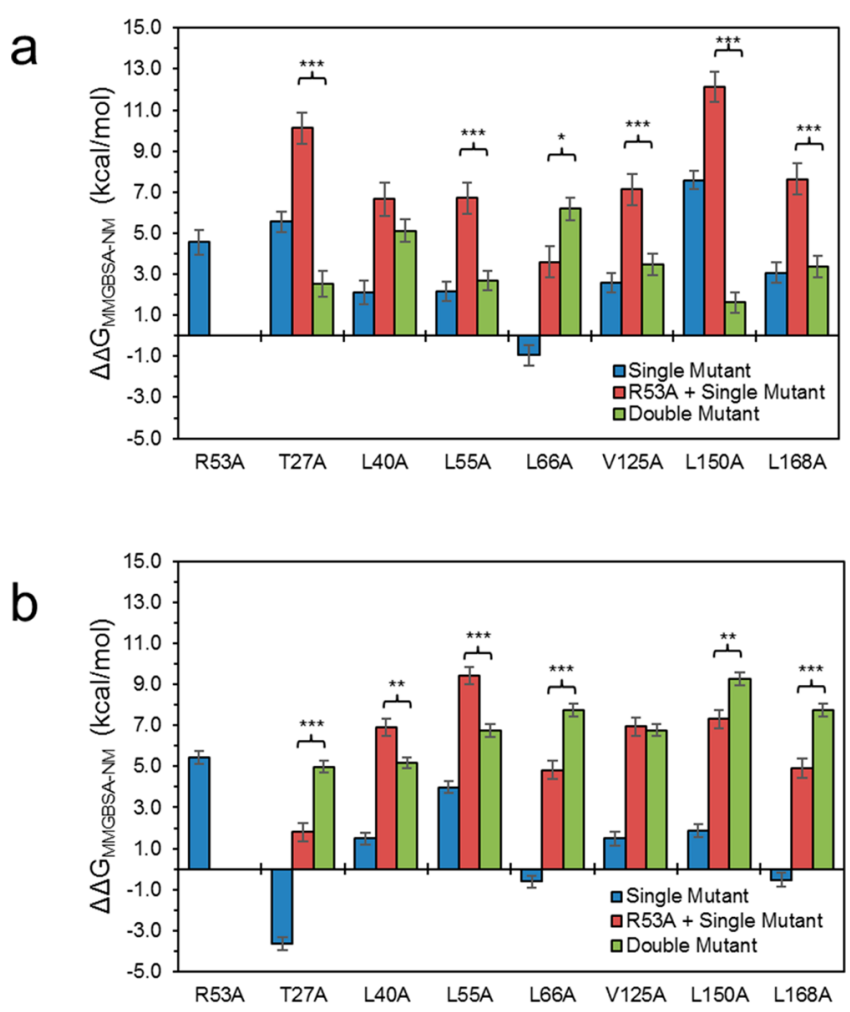

Figure 5. Cooperativity of singly and doubly mutated (a) uPAR.uPA and (b) uPAR 12 complexes. The difference and standard error between the free energy of each mutant with the respective wild-type complex (blue), the sum of the $\Delta \Delta G$ of the Arg-53-Ala mutant and the single mutant (red), and the double mutant (green). Statistical significance (independent two-sample $t$ test): ${ }^{*} p<0.01, * * p<0.001$, and $* * * p<0.0001$.

641 For the uPAR-uPA complex, mutation of all residues except 642 for one (Leu-66) resulted in a penalty in the MM-GBSA free 643 energy consistent with experimental alanine scanning data for 644 these residues (Figure 5a). The sum of the MM-GBSA free 645 energy change as a result of mutation of Arg-53 and each of the 646 amino acids was nearly always unfavorable as shown in Figure 6475 a. However, it is interesting that when both amino acids (Arg64853 and one of the amino acids in Figure 5) were simultaneously 649 mutated, the resulting change in the MM-GBSA free energy 650 was nearly always different than the sum of the individual 651 mutations (Figure 5a). For example, the sum of the individual 652 mutations of Leu-150 and Arg-53 was $12 \mathrm{kcal} \mathrm{mol}^{-1}$, yet the 653 Leu-150-Ala/Arg-153-Ala double mutation resulted in an only $6542 \mathrm{kcal} \mathrm{mol}^{-1}$ change in the free energy of binding. Another 655 interesting example is that of Leu-66, for which the sum of 656 individual mutations of this residue and Arg-153 to alanine was $6574 \mathrm{kcal} \mathrm{mol}^{-1}$, yet the double mutant was much less favorable at 658 nearly $7 \mathrm{kcal} \mathrm{mol}^{-1}$. These results strongly suggest cooperativity 659 between Arg-53 and hot-spot residues toward the binding of $660 \mathrm{uPA}$ to uPAR. For the uPAR-uPA complex, it appears that Arg66153 significantly reduces the size of the contributions of hot 662 spots to the binding of uPA to uPAR.

663 We explored whether Arg-53 and other residues bind to 12 664 in a cooperative manner. In the UPAR 12 complex, mutation of 665 Thr-27, Leu-66, and Leu-168 resulted in more favorable binding, while mutation of the other residues resulted in a 666 loss of affinity of $\mathbf{1 2}$ for uPAR (Figure 5b). Mutation of Arg-53 667 to alanine, as expected, resulted in the most pronounced effect 668 on the binding of $\mathbf{1 2}$ to uPAR. The sum of the contributions of 669 Arg-53 and other residues was unfavorable in nearly every case. 670 Interestingly, double mutants also consistently exhibited an 671 unfavorable effect that was different from the effect of the sum 672 of each mutation. For example, in the case of Thr-27, mutation 673 of this residue along with Arg-53 resulted in a $5 \mathrm{kcal} \mathrm{mol}^{-1} 674$ increase in the free energy of binding compared with only 2675 $\mathrm{kcal} \mathrm{mol}^{-1}$ for the sum of the free energy change of individual 676 mutations. A similar increase was found for Leu-66 and Leu- 677 168. For the other residues, the double mutant showed a 678 change in the free energy less pronounced than the sum of the 679 free energy change for the individual mutations. For example, 680 mutation of Leu-55 and Arg-53 to alanine resulted in a 681 combined free energy change of $9 \mathrm{kcal} \mathrm{mol}^{-1}$, while the Leu-55- 682 $\mathrm{Ala} /$ Arg-53-Ala double mutation resulted in a $7 \mathrm{kcal} \mathrm{mol}^{-1}$ less 683 favorable interaction. Other than for Val-125, Arg-53 exhibited 684 cooperativity with each of the residues that were considered. 685 Interestingly, double mutants with Arg-53 and another residue 686 all resulted in unfavorable energy for both uPAR.uPA and 687 UPAR 12 complexes. Interestingly, for two of the hot spots, 688 Leu-66 and Leu-150, Arg-53 enhances the interaction of 12689 with $\mathrm{uPAR}$ by a combined $5 \mathrm{kcal} \mathrm{mol}^{-1}$, while Arg-53 weakens 690 the interaction of $\mathbf{1 2}$ with hot-spot Leu-55 by $2 \mathrm{kcal} \mathrm{mol}^{-1}$. 691

Small-Molecule UPAR-uPA Inhibitors Alter UPAR 692 Dynamics. The cooperativity among residues prompted us 693 to explore the effect of the two classes of compounds on the 694 dynamics of uPAR. We investigated the correlation of the 695 motion of 3, 12, and several other compounds to each residue 696 on uPAR (Figure 6a). Visual inspection of the color-coded map $697 \mathrm{f} 6$ in Figure 6a reveals a dramatic difference in the correlation 698 profile of compounds. For example, pyrrolinone $\mathbf{1 2}$ and 699 derivatives generally shows strong correlated motion with 700 residues $1-60$ on UPAR, while pyrazole 3 shows no correlation. 701 To compare the effect of $\mathbf{3}$ and $\mathbf{1 2}$ on the dynamics of UPAR 702 with uPA, we generated a cross-correlated map of the motion of 703 $\mathrm{uPAR}$ in the presence of uPA (Figure 6b). Interestingly, the 704 effects of $\mathbf{1 2}$ on the dynamics of UPAR were remarkably similar 705 to those of uPA on uPAR (Figure 6c,e), while 3 (Figure 6d) 706 showed little correlation. These results suggest small molecules 707 that replicate the dynamics of the native ligand in a protein- 708 protein interaction are more likely to disrupt the interaction. 709

\section{DISCUSSION}

Protein-protein interactions range from transient to tight. 711 Transient interactions are weaker micromolar-affinity inter- 712 actions that typically involve simpler binding epitopes such as a 713 linear peptide bound to a cavity. ${ }^{61,62}$ Tight interactions occur 714 between proteins with single- or double-digit nanomolar-range 715 binding constants. Tight interactions involve larger interfaces 716 (1000-3000 $\AA^{2}$ ) with binding epitopes consisting of a single 717 secondary structure such as an $\alpha$ helix, or a more complex 718 binding epitope that spans multiple secondary structures. ${ }^{1,2,63} 719$ While there are numerous examples of small molecules that 720 disrupt weak protein-protein interactions, only a few tight 721 interactions have been successfully inhibited with small 722 molecules. ${ }^{1}$ Despite the smaller footprint of small molecules 723 compared with the large protein-protein interface of tight 724 interactions with complex epitopes, it has been suggested that 725 small molecules disrupt these interactions by engaging hot 726 

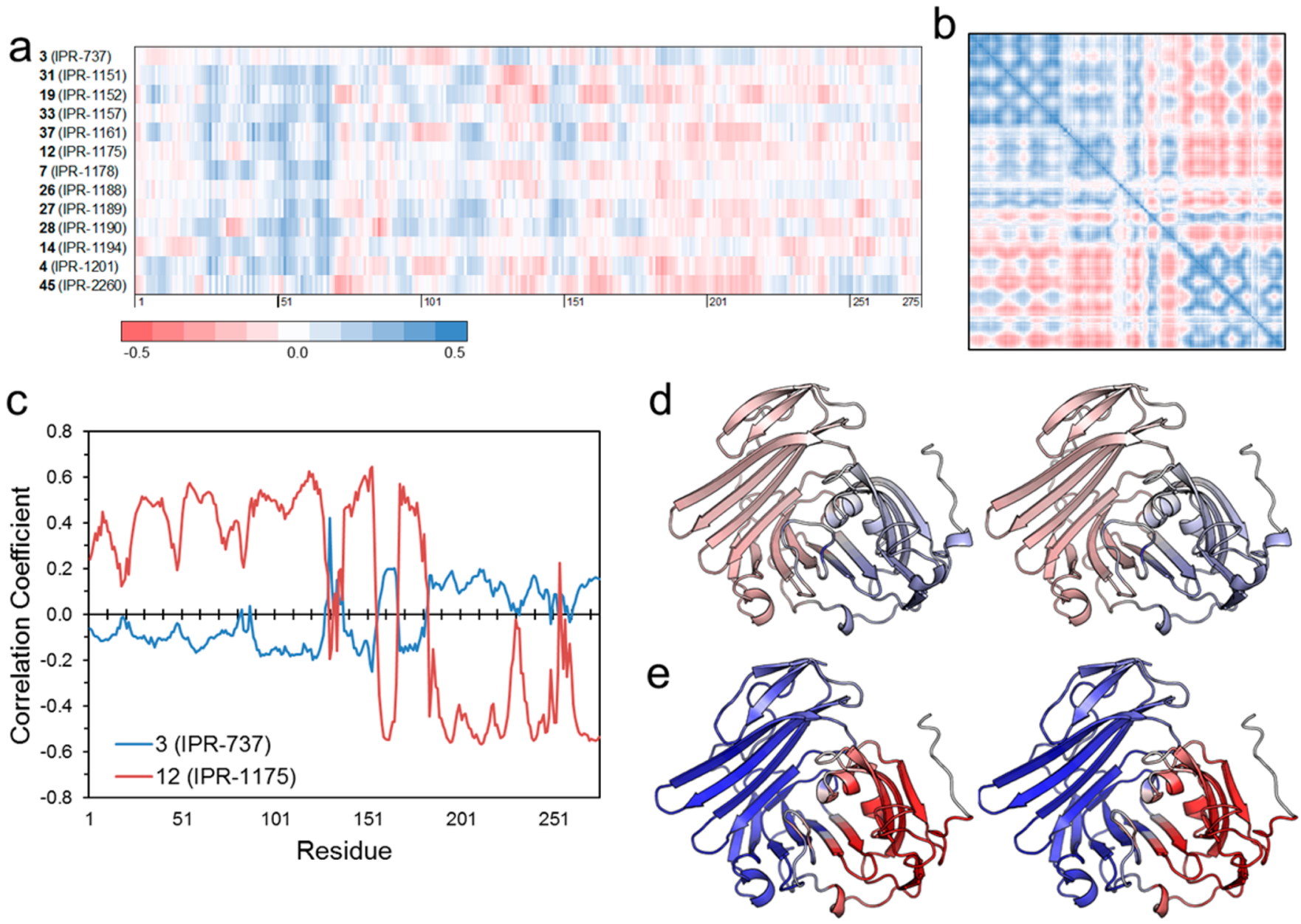

Figure 6. (a) Dynamic cross-correlation matrix (DCCM) cross section of 3 and select derivatives of 1 with uPAR. (b) DCCM of uPAR in the uPAR. uPA complex, colored using the same scheme used for panel a. (c) Pearson's correlation between the cross-correlation of uPAR.3 and uPAR·12 complexes and the cross-correlation of individual residues of uPAR in the uPAR-uPA complex. Stereoviews of three-dimensional structures of uPAR in the (d) uPAR·3 and (e) uPAR 12 complexes, colored on the basis of the correlation coefficient in panel c from positive correlation (blue) to no correlation (white) to negative correlation (red).

727 spots at the interface. ${ }^{9,26,27}$ Hot spots can be located on either 728 the ligand or the receptor binding cleft. ${ }^{5,8,64}$

729 Here, we explore small-molecule antagonists of the uPAR. $730 \mathrm{uPA}$ interaction, a tight and stable protein-protein interaction. 731 The uPAR.uPA interface has a well-defined binding cavity that 732 contains multiple hot-spot residues that contribute $\geq 1 \mathrm{kcal}$ $733 \mathrm{~mol}^{-1}$ to the binding affinity. These hot spots are located on 734 both uPAR and its ligand, uPA. Small molecules that (i) mimic 735 the position of hot spots on the ligand (uPA) and (ii) engage 736 hot spots on the receptor (UPAR) are expected to disrupt this 737 interaction. Previously, we had identified two classes of 738 compounds that disrupt the protein-protein interaction. The 739 first compound, 2, was identified by docking a commercial 740 library to an ensemble of structures of uPAR collected from 741 molecular dynamics simulations. ${ }^{24}$ The compound showed 742 binding affinity in the submicromolar range and an $\mathrm{IC}_{50}$ for 743 disruption of uPAR.uPA interaction in the single-digit micro744 molar range. Interestingly, the predicted mode of binding of 2 745 to a structure of UPAR sampled from molecular dynamics 746 simulation revealed a salt bridge between the benzoic acid 747 moiety of 2 and Arg-53. ${ }^{24}$ A recent crystal structure of an 748 analogue of 2 bound to uPAR confirmed our predicted binding 749 mode of the compound and supported the existence of a salt750 bridge interaction with Arg-53. ${ }^{20}$ Arg-53 is buried in the crystal structure of the uPAR.uPA complex. The snapshot of 2 bound 751 to UPAR that emerged from our molecular dynamics 752 simulations revealed an exposed Arg-53. Interestingly, the apo 753 structure of uPAR, which was determined after we discovered 2, 754 reveals an exposed Arg-53. ${ }^{65}$ This supported our rationale for 755 using molecular dynamics simulations to sample conformations 756 that differ from the structure of UPAR in its complex with uPA. 757 The second class of uPAR $\cdot u P A$ antagonists that we discovered 758 were pyrrolinone compounds. $^{23}$ Interestingly, these com- 759 pounds were identified in a ligand-based approach using the 760 structure of pyrazole, pyrrolodinone, piperidinone, and butan- 761 amine compounds that were identified by structure-based 762 virtual screening using the structure of uPAR from the uPAR 763 uPA complex. ${ }^{21}$ The carbon that bears the hydroxyl group of 764 the pyrrolinone compounds considered in this work may be 765 prone to attack by nucleophiles. However, the $\mathrm{OH}$ group 766 makes a poor leaving group, making covalent adduct formation 767 between the compound and protein unlikely. We confirmed 768 this with mass spectrometry. Further evidence that argues 769 against the formation of a covalent adduct is the fact that 770 replacement of $\mathrm{OH}$ with a methoxy or isopropoxy, each of 771 which is a much better leaving group than $\mathrm{OH}$ is, resulted in 772 lower-affinity compounds. Also, the lack of exposed nucleo- 773 philes such as cysteine residues on uPAR makes it highly 774 
775 unlikely that $\mathbf{1}$ and derivatives covalently attach to uPAR. In 776 addition, we show that the compound is stable in buffer, 777 methanol, and uPAR.

778 We synthesize nearly 50 derivatives of pyrollinone 1 to 779 explore the uPAR.uPA protein-protein interaction. We 780 determined the crystal structure of a derivative of 1 , namely 78112 (IPR-1175), and a pyrazole compound 3 (IPR-737) that 782 binds to uPAR but does not disrupt the uPAR $\cdot \mathrm{uPA}$ interface. 783 The structures reveal that the pyrazole compounds bind to a 784 site on UPAR that is occupied by a UPA side chain located on 785 the $\beta$ strands of the $\beta$ turn of the GFD domain of uPA. The 2786 pyrrolinone compound, however, adopts a different binding 787 mode. In addition, the compound comes in direct contact with 788 several hot spots. This explains why both compounds bind to 789 uPAR but only the 2-pyrrolone inhibits uPAR $\cdot \mathrm{uPA}$ interaction 790 within the $100 \mu \mathrm{M}$ concentration range considered in this 791 work. It was interesting that two of the aromatic rings of $\mathbf{1 2}$ 792 engaged Arg-53, directly forming highly favorable cation $-\pi$ 793 interactions. Cation $-\pi$ interactions are electrostatic interactions 794 that are considered to be among the strongest noncovalent 795 interactions in protein structures, ${ }^{66}$ while mutation of this 796 residue seems to be a common feature of small molecules that 797 we have found to inhibit the uPAR.uPA protein-protein 798 interaction. Previously, we found that a salt-bridge interaction 799 between a benzoic acid moiety on 2 (IPR-803) and the 800 guanidinium ion of Arg-53 was the critical factor that led to 801 inhibition of uPAR.uPA interaction. ${ }^{24}$ Removal of the benzoic 802 acid led to loss of inhibition of the protein-protein interaction. 803 The use of hot spots on UPAR and uPA in future studies is 804 expected to lead to derivatives that are substantially more 805 potent than the parent compound. This could be achieved by 806 enhancing the affinity of compounds to individual hot-spot 807 residues or by mimicking critical hot spots located on uPA, 808 such as Phe- 25 or Trp-30.

809 While Arg-53 is not considered a hot spot, its interaction 810 with small molecules appears to be essential for successful 811 inhibition of the UPAR $\cdot \mathrm{uPA}$ interaction by small molecules. 812 Interestingly, molecular dynamics simulation-based free energy 813 decomposition reveals that Arg-53 makes the strongest 814 interaction with uPA compared with other $\mathrm{UPA}$ residues that 815 come in contact with UPAR. Despite the strong interaction, 816 alanine scanning studies show that Arg-53 only moderately 817 contributes to the binding affinity $\left(0.7 \mathrm{kcal} \mathrm{mol}^{-111}\right)$. This can 818 be explained by the fact that decomposition energies do not 819 take into consideration the configurational entropy contribu820 tions to the binding, in contrast to the alanine scanning that 821 used surface plasmon resonance, which includes both enthalpy 822 and entropy. Considering the position of Arg-53 within the 823 core structure of $\mathrm{UPAR}$, it is possible that mutation of Arg-53 824 may have destabilized UPAR and hence enhanced entropy 825 contributions to the binding affinity, despite the significant loss 826 of enthalpy. Small-molecule inhibitors are expected to disrupt 827 only the interaction of residues and not their contributions to 828 the entropy of binding. This suggests that residues that are not 829 considered hot spots should not be ignored in the design of 830 small-molecule protein-protein interaction inhibitors.

831 The discovery of hot spots at protein-protein interfaces was 832 a significant breakthrough in the field of protein-protein 833 interaction drug discovery. The primary tools to identify hot 834 spots is to systematically mutate each residue to alanine and 835 measure the effect on the binding thermodynamics and kinetics. 836 This process is known as alanine scanning. One limitation of 837 these alanine scanning experiments is that they do not consider the effects of neighboring residues, which may enhance or 838 weaken the contributions of individual residues. ${ }^{67,68}$ How to 839 take advantage of hot spots for small-molecule drug discovery is 840 still unclear. To gain deeper insight into the role of Arg-53 in 841 the binding of the small-molecule inhibitors, we explored 842 whether any cooperativity between the residue and other 843 residues that come in contact with 1 exists. Interestingly, we 844 found that the independent mutation of Arg-53 and other hot 845 spots resulted in changes in the predicted binding affinity that 846 were different from those measured when both Arg-53 and hot 847 spots were mutated. For uPA, mutation of hot spots resulted in 848 a less favorable MM-GBSA free energy for six of the seven 849 mutations, yet mutations of Arg-53 and each of these residues 850 resulted in dramatically different free energy changes. For 851 example, the mutation of Arg-53 and Leu-150 led to a 852 combined $13 \mathrm{kcal} \mathrm{mol}^{-1}$ MM-GBSA free energy change, yet the 853 combined mutation of these two residues resulted in a change 854 of only $2 \mathrm{kcal} \mathrm{mol}^{-1}$, a dramatic difference. For compound 12, 855 mutation of Arg-53 and Leu-150 led to MM-GBSA free energy 856 changes of 6 and $2 \mathrm{kcal} \mathrm{mol}^{-1}$, respectively. This is in contrast 857 to the dual mutation of both residues that resulted in an 858 unfavorable change of $>9 \mathrm{kcal} \mathrm{mol}^{-1}$.

859

In summary, our work provides insight into the forces that 860 drive small molecules to disrupt tight and stable protein- 861 protein interactions. We show that mere binding is not a 862 sufficient requirement for disrupting the uPAR.uPA protein- 863 protein interaction. Even binding to hot-spot residues is not a 864 guarantee of disruption of the protein-protein interaction. We 865 find that residues that are not considered hot spots in alanine 866 scanning experiments such as Arg-53 in uPAR can provide a 867 strong anchor for small molecules to enhance their binding 868 affinity. These anchors can also strengthen binding of small 869 molecules to other hot spots through cooperativity. Also, salt- 870 bridge and $\pi$-cation interactions appear to be critical for 871 disruption of the uPAR.uPA interaction. A previously 872 discovered UPAR antagonist, IPR-803, formed a salt-bridge 873 interaction that was essential for inhibition of uPAR.uPA 874 interaction, ${ }^{24}$ and compounds reported in this work bind to 875 Arg-53 through $\pi$-cation interactions. These types of 876 interactions are likely to be favorable at protein-protein 877 interfaces considering the shallow nature of the pockets and the 878 high degree of solvent exposure. These interactions may be a 879 straightforward strategy for enhancing the inhibition potency of 880 compounds that bind to the target. Another important finding 881 of this work is the importance of molecular dynamics in the 882 inhibition of tight PPIs by small molecules. We discovered that 883 small molecules that inhibit uPAR-uPA interaction alter the 884 dynamics of uPAR in a manner similar to that of uPA. This 885 suggests that drug discovery efforts targeting protein-protein 886 interactions should also consider the effect of compounds on 887 the dynamics of the receptor as opposed to merely focusing on 888 the interaction energy. Small molecules that bind tightly to hot 889 spots and also modulate the dynamics of the receptor in a 890 manner similar to that of the native tight ligand are expected to 891 lead to more effective inhibitors. These findings can help guide 892 the design of derivatives of IPR-1110 through modification of 893 the core structure of the compound or by the introduction of 894 new substituents. 


\section{ASSOCIATED CONTENT}

\section{S Supporting Information}

898 The Supporting Information is available free of charge on the 899 ACS Publications website at DOI: 10.1021/acs.bio900 chem.6b01039.

901 Chemical structures of compounds that emerged from 902 virtual screening against the UPAR crystal structure and 903 multiple conformers, hot spots on uPAR of the uPAR. 904 uPA interaction, adapted from ref 18 (Table S1), X-ray 905 data collection and refinement statistics of the suPARcc. 90612 (IPR-1175) complex (Table S2), X-ray data collection 907 and refinement statistics of the suPARcc.3 (IPR-737) 908 complex (Table S3), calculated free energies \pm the 909 standard error of $\mathbf{3}$ and select derivatives of $\mathbf{1}$, showing 910 the individual free energy terms (Table S4), correlation 911 coefficients of the individual free energy terms and 912 correlation to select residues of UPAR to illustrate the 913 relationship between the decomposition energy and 914 inhibition constant $K_{\mathrm{i}}$ for select derivatives of $\mathbf{1}$ from 915 Figure 4c (Table S5), correlation coefficients to illustrate 916 the relationship between the decomposition energy of 917 select residues from Figure $4 \mathrm{c}$ and kinetic rate constants 918 for select derivatives of $\mathbf{1}$ (Table S6), and calculated free 919 energies \pm the standard error of single and double 920 mutants in the uPAR.uPA and uPAR.12 complexes 921 (Table S7) (PDF)

\section{$922 \square$ AUTHOR INFORMATION}

\section{Corresponding Author}

924 * Departments of Biochemistry and Molecular Biology, Indiana 925 University School of Medicine, 410 W. 10th St., HITS 5000, 926 Indianapolis, IN 46202. E-mail: smeroueh@iu.edu. Telephone: 927 (317) 274-8315. Fax: (317) 278-9217.

\section{ORCID}

929 Mingdong Huang: 0000-0002-1377-6786

930 Samy O. Meroueh: 0000-0002-3948-7499

\section{Author Contributions}

932 D.L. and D.X. contributed equally to this work.

\section{Funding}

934 The research was supported by the National Institutes of 935 Health (CA135380) (S.O.M.), American Cancer Society 936 Research Scholar Grant RSG-12-092-01-CDD (S.O.M.), and 937 the 100 Voices of Hope (S.O.M.). Computer time on the Big 938 Red II and Karst supercomputer at Indiana University (S.O.M.) 939 is supported in part by Lilly Endowment, Inc., through its 940 support for the Indiana University Pervasive Technology 941 Institute, and in part by the Indiana METACyt Initiative.

942 Notes

943 The authors declare no competing financial interest.

\section{REFERENCES}

945 (1) Arkin, M. R., Tang, Y., and Wells, J. A. (2014) Small-Molecule 946 Inhibitors of Protein-Protein Interactions: Progressing Toward the 947 Reality. Chem. Biol. 21, 1102-1114.

948 (2) Watkins, A. M., and Arora, P. S. (2015) Structure-based 949 inhibition of protein-protein interactions. Eur. J. Med. Chem. 94, 480950488.

951 (3) Oltersdorf, T., Elmore, S. W., Shoemaker, A. R., Armstrong, R. 952 C., Augeri, D. J., Belli, B. A., Bruncko, M., Deckwerth, T. L., Dinges, J., 953 Hajduk, P. J., et al. (2005) An inhibitor of Bcl-2 family proteins 954 induces regression of solid tumours. Nature 435, 677-681.
(4) Wilson, C. G., and Arkin, M. R. (2013) Probing structural 955 adaptivity at PPI interfaces with small molecules. Drug Discovery 956 Today: Technol. 10, e501-508.

(5) Cukuroglu, E., Engin, H. B., Gursoy, A., and Keskin, O. (2014) 958 Hot spots in protein-protein interfaces: towards drug discovery. Prog. 959 Biophys. Mol. Biol. 116, 165-173.

960

(6) Kortemme, T., and Baker, D. (2002) A simple physical model for 961 binding energy hot spots in protein-protein complexes. Proc. Natl. 962 Acad. Sci. U. S. A. 99, 14116-14121.

(7) Clackson, T., and Wells, J. A. (1995) A Hot Spot of Binding 964 Energy in a Hormone-Receptor Interface. Science 267, 383-386. 965

(8) Keskin, O., Ma, B., and Nussinov, R. (2005) Hot regions in 966 protein-protein interactions: the organization and contribution of 967 structurally conserved hot spot residues. J. Mol. Biol. 345, 1281-1294. 968

(9) Bogan, A. A., and Thorn, K. S. (1998) Anatomy of Hot Spots in 969 Protein Interfaces. J. Mol. Biol. 280, 1-9.

(10) Magdolen, V., Rettenberger, P., Koppitz, M., Goretzkt, L., 971 Kessler, H., Weidle, U. H., Konig, B., Graeff, H., Schmitt, M., and 972 Wilhelm, O. (1996) Systematic Mutational Analysis of the Receptor- 973 Binding Region of the Human Urokinase-Type Plasminogen Activator. 974 Eur. J. Biochem. 237, 743-751.

(11) Gardsvoll, H., and Ploug, M. (2007) Mapping of the vitronectin- 976 binding site on the urokinase receptor: involvement of a coherent 977 receptor interface consisting of residues from both domain I and the 978 flanking interdomain linker region. J. Biol. Chem. 282, 13561-13572. 979

(12) Madsen, C. D., Ferraris, G. M., Andolfo, A., Cunningham, O., 980 and Sidenius, N. (2007) uPAR-induced cell adhesion and migration: 981 vitronectin provides the key. J. Cell Biol. 177, 927-939.

982

(13) Huai, Q., Zhou, A., Lin, L., Mazar, A. P., Parry, G. C., Callahan, 983 J., Shaw, D. E., Furie, B., Furie, B. C., and Huang, M. (2008) Crystal 984 structures of two human vitronectin, urokinase and urokinase receptor 985 complexes. Nat. Struct. Mol. Biol. 15, 422-423.

986

(14) Lin, L., Gardsvoll, H., Huai, Q., Huang, M., and Ploug, M. 987 (2010) Structure-based engineering of species selectivity in the 988 interaction between urokinase and its receptor: implication for 989 preclinical cancer therapy. J. Biol. Chem. 285, 10982-10992. 990

(15) Gardsvoll, H., Dano, K., and Ploug, M. (1999) Mapping part of 991 the functional epitope for ligand binding on the receptor for urokinase- 992 type plasminogen activator by site-directed mutagenesis. J. Biol. Chem. 993 274, 37995-38003.

(16) Huai, Q., Mazar, A. P., Kuo, A., Parry, G. C., Shaw, D. E., 995 Callahan, J., Li, Y., Yuan, C., Bian, C., and Chen, L. (2006) Structure of 996 human urokinase plasminogen activator in complex with its receptor. 997 Science 311, 656-659.

998

(17) Llinas, P., Hélène Le Du, M., Gardsvoll, H., Dano, K., Ploug, M., 999 Gilquin, B., Stura, E. A., and Menez, A. (2005) Crystal structure of the 1000 human urokinase plasminogen activator receptor bound to an 1001 antagonist peptide. EMBO J. 24, 1655-1663.

1002

(18) Gardsvoll, H., Gilquin, B., Le Du, M. H., Menez, A., Jorgensen, 1003 T. J., and Ploug, M. (2006) Characterization of the functional epitope 1004 on the urokinase receptor. Complete alanine scanning mutagenesis 1005 supplemented by chemical cross-linking. J. Biol. Chem. 281, 19260- 1006 19272.

1007

(19) Chen, Z., Lin, L., Huai, Q., and Huang, M. (2009) Challenges 1008 for drug discovery-a case study of urokinase receptor inhibition. Comb. 1009 Chem. High Throughput Screening 12, 961.

1010

(20) Mani, T., Wang, F., Knabe, W. E., Sinn, A. L., Khanna, M., Jo, I., 1011 Sandusky, G. E., Sledge, G. W., Jr., Jones, D. R., Khanna, R., Pollok, K. 1012 E., and Meroueh, S. O. (2013) Small-molecule inhibition of the 1013 uPAR.uPA interaction: synthesis, biochemical, cellular, in vivo 1014 pharmacokinetics and efficacy studies in breast cancer metastasis. 1015 Bioorg. Med. Chem. 21, 2145-2155.

1016

(21) Mani, T., Liu, D., Zhou, D., Li, L., Knabe, W. E., Wang, F., Oh, 1017 K., and Meroueh, S. O. (2013) Probing binding and cellular activity of 1018 pyrrolidinone and piperidinone small molecules targeting the 1019 urokinase receptor. ChemMedChem 8, 1963-1977.

1020

(22) Wang, F., Eric Knabe, W., Li, L., Jo, I., Mani, T., Roehm, H., Oh, 1021 K., Li, J., Khanna, M., and Meroueh, S. O. (2012) Design, synthesis, 1022 biochemical studies, cellular characterization, and structure-based 1023 
1024 computational studies of small molecules targeting the urokinase 1025 receptor. Bioorg. Med. Chem. 20, 4760-4773.

1026 (23) Liu, D., Zhou, D., Wang, B., Knabe, W. E., and Meroueh, S. O. 1027 (2015) A New Class of Orthosteric uPAR.uPA Small-Molecule 1028 Antagonists Are Allosteric Inhibitors of the uPAR.Vitronectin 1029 Interaction. ACS Chem. Biol. 10, 1521-1534.

1030 (24) Khanna, M., Wang, F., Jo, I., Knabe, W. E., Wilson, S. M., Li, L., 1031 Bum-Erdene, K., Li, J., Sledge, G. W., Khanna, R., and Meroueh, S. O. 1032 (2011) Targeting multiple conformations leads to small molecule 1033 inhibitors of the uPAR.uPA protein-protein interaction that block 1034 cancer cell invasion. ACS Chem. Biol. 6, 1232-1243.

1035 (25) Rullo, A. F., Fitzgerald, K. J., Muthusamy, V., Liu, M., Yuan, C., 1036 Huang, M., Kim, M., Cho, A. E., and Spiegel, D. A. (2016) Re1037 engineering the Immune Response to Metastatic Cancer: Antibody1038 Recruiting Small Molecules Targeting the Urokinase Receptor. Angew. 1039 Chem., Int. Ed. 55, 3642.

1040 (26) Kortemme, T., and Baker, D. (2002) A simple physical model 1041 for binding energy hot spots in protein-protein complexes. Proc. Natl. 1042 Acad. Sci. U. S. A. 99, 14116-14121.

1043 (27) Clackson, T., and Wells, J. A. (1995) A hot spot of binding 1044 energy in a hormone-receptor interface. Science 267, 383-386.

1045 (28) Thanos, C. D., DeLano, W. L., and Wells, J. A. (2006) Hot-Spot 1046 Mimicry of a Cytokine Receptor by a Small Molecule. Proc. Natl. Acad. 1047 Sci. U. S. A. 103, 15422-15427.

1048 (29) Hyde, J., Braisted, A. C., Randal, M., and Arkin, M. R. (2003) 1049 Discovery and characterization of cooperative ligand binding in the 1050 adaptive region of interleukin-2. Biochemistry 42, 6475-6483.

1051 (30) Keskin, Z., Gursoy, A., Ma, B., and Nussinov, R. (2008) 1052 Principles of protein-protein interactions: What are the preferred ways 1053 for proteins to interact? Chem. Rev. 108, 1225-1244.

1054 (31) Jacobsen, B., Gårdsvoll, H., Juhl Funch, G., Ostergaard, S., 1055 Barkholt, V., and Ploug, M. (2007) One-step affinity purification of 1056 recombinant urokinase-type plasminogen activator receptor using a 1057 synthetic peptide developed by combinatorial chemistry. Protein 1058 Expression Purif. 52, 286-296.

1059 (32) Xu, X., Gardsvoll, H., Yuan, C., Lin, L., Ploug, M., and Huang, 1060 M. (2012) Crystal structure of the urokinase receptor in a ligand-free 1061 form. J. Mol. Biol. 416, 629-641.

1062 (33) Otwinowski, Z., and Minor, W. (1997) Processing of X-ray 1063 diffraction data collected in oscillation mode. Methods Enzymol. 276, 1064 307-326.

1065 (34) Vagin, A., and Teplyakov, A. (1997) MOLREP: an Automated 1066 Program for Molecular Replacement. J. Appl. Crystallogr. 30, 102210671025.

1068 (35) Emsley, P., and Cowtan, K. (2004) Coot: model-building tools 1069 for molecular graphics. Acta Crystallogr., Sect. D: Biol. Crystallogr. 60, $10702126-2132$.

1071 (36) Jacobson, M. P., Pincus, D. L., Rapp, C. S., Day, T. J., Honig, B., 1072 Shaw, D. E., and Friesner, R. A. (2004) A Hierarchical Approach to 1073 All-Atom Protein Loop Prediction. Proteins: Struct., Funct., Genet. 55, 1074 351-367.

1075 (37) Sali, A., and Blundell, T. L. (1993) Comparative Protein 1076 Modelling by Satisfaction of Spatial Restraints. J. Mol. Biol. 234, 7791077815.

1078 (38) Olsson, M. H. M., Søndergaard, C. R., Rostkowski, M., and 1079 Jensen, J. H. (2011) PROPKA3: Consistent Treatment of Internal and 1080 Surface Residues in Empirical pKa Predictions. J. Chem. Theory 1081 Comput. 7, 525-537.

1082 (39) Halgren, T. A., Murphy, R. B., Friesner, R. A., Beard, H. S., Frye, 1083 L. L., Pollard, W. T., and Banks, J. L. (2004) Glide: a new approach for 1084 rapid, accurate docking and scoring. 2. Enrichment factors in database 1085 screening. J. Med. Chem. 47, 1750-1759.

1086 (40) Case, D. A., Berryman, J. T., Betz, R. M., Cerutti, D. S., 1087 Cheatham, T. E., III, Darden, T. A., Duke, R. E., Giese, T. J., Gohlke, 1088 H., Goetz, A. W., Homeyer, N., Izadi, S., Janowski, P., Kaus, J., 1089 Kovalenko, A., Lee, T. S., LeGrand, S., Li, P., Luchko, T., Luo, R., 1090 Madej, B., Merz, K. M., Monard, G., Needham, P., Nguyen, H., 1091 Nguyen, H. T., Omelyan, I., Onufriev, A., Roe, D. R., Roitberg, A., 1092 Salomon-Ferrer, R., Simmerling, C. L., Smith, W., Swails, J., Walker, R.
C., Wang, J., Wolf, R. M., Wu, X., York, D. M., and Kollman, P. A. 1093 (2015) AMBER 2015, University of California, San Francisco. 1094 (41) Jakalian, A., Jack, D. B., and Bayly, C. I. (2002) Fast, Efficient 1095 Generation of High-quality Atomic Charges. AM1-BCC Model: II. 1096 Parameterization and Validation. J. Comput. Chem. 23, 1623-1641. 1097

(42) Wang, J., Wolf, R. M., Caldwell, J. W., Kollman, P. A., and Case, 1098 D. A. (2004) Development and Testing of a General Amber Force 1099 Field. J. Comput. Chem. 25, 1157-1174.

1100

(43) Wang, J., Wang, W., Kollman, P. A., and Case, D. A. (2006) 1101 Automatic Atom Type and Bond Type Perception in Molecular 1102 Mechanical Calculations. J. Mol. Graphics Modell. 25, 247-260. 1103

(44) Jorgensen, W. L., Chandrasekhar, J., Madura, J. D., Impey, R. 1104 W., and Klein, M. L. (1983) Comparison of Simple Potential 1105 Functions for Simulating Liquid Water. J. Chem. Phys. 79, 926-935. 1106

(45) Maier, J. A., Martinez, C., Kasavajhala, K., Wickstrom, L., 1107 Hauser, K. E., and Simmerling, C. (2015) ff14SB: Improving the 1108 Accuracy of Protein Side Chain and Backbone Parameters from 1109 ff99SB. J. Chem. Theory Comput. 11, 3696-3713. 1110

(46) Ryckaert, J. P., Ciccotti, G., and Berendsen, J. J. C. (1977) 1111 Numerical Integration of the Cartesian Equations of Motion of a 1112 System with Constraints: Molecular Dynamics of n-Alkanes. J. Comput. 1113 Phys. 23, 327-341.

1114

(47) Darden, T., York, D., and Pedersen, L. (1993) Particle Mesh 1115 Ewald: An $\mathrm{N} \times \log (\mathrm{N})$ Method for Ewald Sums in Large Systems. J. 1116 Chem. Phys. 98, 10089-10092.

(48) Roe, D. R., and Cheatham, T. E., 3rd (2013) PTRAJ and 1118 CPPTRAJ: Software for Processing and Analysis of Molecular 1119 Dynamics Trajectory Data. J. Chem. Theory Comput. 9, 3084-3095. 1120 (49) Still, W. C., Tempczyk, A., Hawley, R. C., and Hendrickson, T. 1121 (1990) Semianalytical Treatment of Solvation for Molecular 1122 Mechanics and Dynamics. J. Am. Chem. Soc. 112, 6127-6129. 1123

(50) Miller, B. R., 3rd, McGee, T. D., Jr., Swails, J. M., Homeyer, N., 1124 Gohlke, H., and Roitberg, A. E. (2012) MMPBSA.py: An Efficient 1125 Program for End-State Free Energy Calculations. J. Chem. Theory 1126 Comput. 8, 3314-3321.

(51) Onufriev, A., Bashford, D., and Case, D. A. (2004) Exploring 1128 Protein Native States and Large-scale Conformational Changes with a 1129 Modified Generalized Born Model. Proteins: Struct., Funct., Genet. 55, 1130 383-394.

1131

(52) Feig, M., Onufriev, A., Lee, M. S., Im, W., Case, D. A., and 1132 Brooks, C. L., 3rd (2004) Performance Comparison of Generalized 1133 Born and Poisson Methods in the Calculation of Electrostatic 1134 Solvation Energies for Protein Structures. J. Comput. Chem. 25, 1135 265-284.

1136

(53) Brooks, B., and Karplus, M. (1983) Harmonic Dynamics of 1137 Proteins: Normal Modes and Fluctuations in Bovine Pancreatic 1138 Trypsin Inhibitor. Proc. Natl. Acad. Sci. U. S. A. 80, 6571-6575. 1139

(54) Gohlke, H., Kiel, C., and Case, D. A. (2003) Insights into 1140 Protein-Protein Binding by Binding Free Energy Calculation and 1141 Free Energy Decomposition for the Ras-Raf and Ras-RalGDS 1142 Complexes. J. Mol. Biol. 330, 891-913.

1143

(55) Onufriev, A., Bashford, D., and Case, D. A. (2004) Exploring 1144 Protein Native States and Large-scale Conformational Changes with a 1145 Modified Generalized Born Model. Proteins: Struct., Funct., Genet. 55, 1146 383-394.

1147

(56) De Marco, A., De Candia, M., Carotti, A., Cellamare, S., De 1148 Candia, E., and Altomare, C. (2004) Lipophilicity-related inhibition of 1149 blood platelet aggregation by nipecotic acid anilides. Eur. J. Pharm. Sci. 1150 22, 153-164.

(57) Milagre, C. D., Milagre, H. M., Moran, P. J., and Rodrigues, J. A. 1152 (2010) Chemoenzymatic synthesis of alpha-hydroxy-beta-methyl- 1153 gamma-hydroxy esters: role of the keto-enol equilibrium to control 1154 the stereoselective hydrogenation in a key step. J. Org. Chem. 75, 1155 $1410-1418$.

1156

(58) Richter, A., Rose, R., Hedberg, C., Waldmann, H., and Ottmann, 1157 C. (2012) An optimized small-molecule stabiliser of the 14-3-3- 1158 PMA2 protein-protein interaction. Chem. - Eur. J. 18, 6520-6527. 1159 
1160 (59) Mahadevi, A. S., and Sastry, G. N. (2013) Cation-pi interaction: 1161 its role and relevance in chemistry, biology, and material science. 1162 Chem. Rev. 113, 2100-2138.

1163 (60) Mecozzi, S., West, A. P., and Dougherty, D. A. (1996) Cation-pi 1164 interactions in aromatics of biological and medicinal interest: 1165 electrostatic potential surfaces as a useful qualitative guide. Proc. 1166 Natl. Acad. Sci. U. S. A. 93, 10566-10571.

1167 (61) Nooren, I. M. A., and Thornton, J. M. (2003) Structural 1168 characterisation and functional significance of transient protein-protein 1169 interactions. J. Mol. Biol. 325, 991-1018.

1170 (62) Acuner Ozbabacan, S. E., Engin, H. B., Gursoy, A., and Keskin, 1171 O. (2011) Transient protein-protein interactions. Protein Eng., Des. 1172 Sel. 24, 635-648.

1173 (63) Pelay-Gimeno, M., Glas, A., Koch, O., and Grossmann, T. N. 1174 (2015) Structure-Based Design of Inhibitors of Protein-Protein 1175 Interactions: Mimicking Peptide Binding Epitopes. Angew. Chem., Int. 1176 Ed. 54, 8896-8927.

1177 (64) Cukuroglu, E., Gursoy, A., and Keskin, O. (2012) HotRegion: a 1178 database of predicted hot spot clusters. Nucleic Acids Res. 40, D8291179833.

1180 (65) Xu, X., Gårdsvoll, H., Yuan, C., Lin, L., Ploug, M., and Huang, 1181 M. (2012) Crystal structure of the urokinase receptor in a ligand-free 1182 form. J. Mol. Biol. 416, 629-641.

1183 (66) Dougherty, D. A. (2013) The cation $-\pi$ interaction. Acc. Chem. 1184 Res. 46, 885-893.

1185 (67) Vaughan, C. K., Buckle, A. M., and Fersht, A. R. (1999) 1186 Structural response to mutation at a protein-protein interface. J. Mol. 1187 Biol. 286, 1487-1506.

1188 (68) Schreiber, G., and Fersht, A. R. (1995) Energetics of protein1189 protein interactions: analysis of the barnase-barstar interface by single 1190 mutations and double mutant cycles. J. Mol. Biol. 248, 478-486. 